\title{
The Double Star magnetic field investigation: instrument design, performance and highlights of the first year's observations
}

\author{
C. Carr ${ }^{1}$, P. Brown ${ }^{1}$, T. L. Zhang ${ }^{2}$, J. Gloag ${ }^{1}$, T. Horbury ${ }^{1}$, E. Lucek ${ }^{1}$, W. Magnes ${ }^{2}$, H. O'Brien ${ }^{1}$, T. Oddy ${ }^{1}$, U. Auster ${ }^{3}$, \\ P. Austin ${ }^{4}$, O. Aydogar ${ }^{2}$, A. Balogh ${ }^{1}$, W. Baumjohann ${ }^{2}$, T. Beek ${ }^{1}$, H. Eichelberger ${ }^{2}$, K.-H. Fornacon ${ }^{3}$, E. Georgescu ${ }^{5}$, \\ K.-H. Glassmeier ${ }^{3}$, M. Ludlam ${ }^{1}$, R. Nakamura ${ }^{2}$, and I. Richter ${ }^{3}$ \\ ${ }^{1}$ The Blackett Laboratory, Imperial College London SW7 2BW, UK \\ ${ }^{2}$ Space Research Institute, Austrian Academy of Sciences, A-8042 Graz, Austria \\ ${ }^{3}$ Institut für Geophysik und extraterrestrische Physik, D-38106 Braunschweig, Germany \\ ${ }^{4}$ Ultra Electronics Signature Management Systems Ltd, Hednesford WS12 5QZ, UK \\ ${ }^{5}$ Max-Planck-Institut für extraterrestrische Physik, D-85748 Garching, Germany
}

Received: 18 February 2005 - Revised: 2 August 2005 - Accepted: 18 April 2005 - Published: 8 November 2005

Part of Special Issue "Double Star - First Results"

\begin{abstract}
One of the primary objectives of the Double Star mission is the accurate measurement of the magnetic field vector along the orbits of the two spacecraft. The magnetic field is an essential parameter for the understanding of space plasma processes and is also required for the effective interpretation of data from the other instruments on the spacecraft. We present the design of the magnetic field instrument onboard both of the Double Star spacecraft and an overview of the performance as measured first on-ground and then inorbit. We also report the results of in-flight calibration of the magnetometers, and the processing methods employed to produce the final data products which are provided to Double Star investigators, and the wider community in general. Particular attention is paid to the techniques developed for removing magnetic interference generated by the solar arrays on the first (equatorial orbiting) spacecraft. Results from the first year of operations are reviewed in the context of combined observations by Double Star and Cluster, and examples given from the different regions visited by the spacecraft to date.
\end{abstract}

Keywords. Magnetospheric physics (Instruments and techniques) - Space plasma physics (Experimental and mathematical techniques, Instruments and techniques)

\section{Introduction}

Knowledge of the local magnetic field is an essential requirement for the study of space plasmas. Most plasma phenomena are ordered with respect to the magnetic field, and consequently they are primarily recognised through magnetic

Correspondence to: C. Carr

(c.m.carr@imperial.ac.uk) field observations. Boundaries between plasmas of different origins, such as the magnetopause, are formed of thin current sheets which are readily identified by their magnetic signature. Wave and particle phenomena are also strongly ordered by the underlying magnetic field. In the context of a space plasma mission, the magnetic field is of intrinsic interest, and also is essential for the interpretation of data from wave and particle experiments. The accurate measurement of the magnetic field along the orbits of the two Double Star spacecraft is therefore a primary objective of the mission. The Double Star Magnetic Field Investigation (FGM Instrument) has the fundamental task to determine the magnetic field vector in the range DC to $10 \mathrm{~Hz}$, with the highest possible accuracy and over as much of the orbit-track as possible. A corollary to this is that the instrument must perform continuously and reliably, which requires an inherent ruggedness of design and fault-tolerance.

Double Star is a magnetospheric physics mission of the Chinese National Space Administration. Operationally, the two Double Star spacecraft TC-1 and TC-2 have been specifically designed to complement the European Space Agency's four-spacecraft Cluster mission. Together, the constellation of Cluster-Double Star offers the possibility of simultaneous multi-point, multi-scale observations which greatly enhance the scope of topics which can be addressed by either mission on its own. Furthermore, the specific synchronisation of the Cluster and Double Star orbits offers the possibility to make repeated and regular multi-point measurements in regions where this has not before been possible (Liu et al., 2005).

The TC- 1 spacecraft is in a highly eccentric orbit inclined at $28^{\circ}$ to the equator, whilst TC- 2 is in a polar orbit. The geocentric apogee distances are $13.4 R_{E}$ and $7 R_{E}$ respectively. Perigee altitude is $570 \mathrm{~km}$ for TC- 1 and $700 \mathrm{~km}$ for 
Table 1. Double Star magnetic field investigator team.

\begin{tabular}{|c|c|}
\hline PI, TC-1 FGM instrument & C. Carr (Imperial College London) \\
\hline PI, TC-2 FGM instrument & T. L. Zhang (IWF Graz) \\
\hline Imperial College London & A. Balogh, P. Brown, P. Cargill, T. Horbury, E. Lucek \\
\hline IWF Graz & W. Baumjohann, W. Magnes, R. Nakamura, K. Schwingenschuh, K. Torkar \\
\hline IGM TU-Braunschweig & U. Auster, K.-H. Glassmeier, K.-H. Fornacon, I. Richter, U. Motschmann \\
\hline CSSAR Beijing, Chinese Academy of Sciences & T. Chen, C. Shen, C. Wang, N. Wang, H. Zhao, Q. Zong \\
\hline Peking University & $\mathrm{S} . \mathrm{Fu}, \mathrm{Z} . \mathrm{Pu}$ \\
\hline Inst. of Geo., Chinese Academy of Sciences & W. Xu \\
\hline University of Science and Technology of China & Y. Hu, S. Jin \\
\hline Wuhan University & X. Deng \\
\hline Finnish Meteorological Institute & O. Amm \\
\hline MPE Garching & E. Georgescu \\
\hline International University Bremen & J. Vogt \\
\hline Rutherford Appleton Laboratory & M. Dunlop \\
\hline St. Petersburg State University & V. Sergeev \\
\hline CETP Velizy & N. Cornilleau-Wehrlin \\
\hline CESR Toulouse & H. Rème \\
\hline MSSL / UCL & A. Fazakerley \\
\hline National University of Ireland Maynooth & S. McKenna-Lawlor \\
\hline University of Sheffield & H. Alleyne \\
\hline
\end{tabular}

TC-2. The TC-1 dayside orbit reaches to the magnetopause, and frequently also beyond the bow-shock, and when apogee is on the night-side then it is in the near-Earth tail region. Double Star offers the possibility to study the sub-solar magnetopause on the dayside, and six months later the currentdisruption region of the magnetotail. By synchronising the orbits with Cluster (Figs. 14 and 17), additional possibilities become apparent. For example, when TC-1 is in the current disruption region then Cluster can be looking for nearEarth neutral line reconnection further down the tail. This is the first time that spacecraft have been in these two regions simultaneously, and this offers the possibility to measure which of these two processes - current disruption or tail reconnection - comes first during a sub-storm. Similarly on the dayside, TC- 1 can look for reconnection at the magnetopause, while both Cluster and TC-2 are in the cusp region (at differing altitudes). This provides the opportunity to test theories of how energy from reconnection is propagated from the reconnection site into the magnetosphere and down to the ionosphere.

The Cluster-Double Star collaboration extends also to the scientific instrumentation onboard the six spacecraft, since most of the Double Star instruments are either flightspare instruments from Cluster or new units built to the same specification. The magnetometer bears direct heritage from the Cluster instrument (Balogh et al., 1993, 1997, 2001), though a substantial instrument re-design was required. There were two reasons for this: firstly, the Cluster instrument contains certain US manufactured components which could not be included in a mission launched from China, and secondly, the instrument had to be provided for both spacecraft (while there is only one Cluster flightspare experiment). Consequently, the magnetometer team re-organised and determined to produce a new instrument with the same form, fit and function as the Cluster original. The hardware team comprises the Space \& Atmospheric Physics Group of Imperial College London, the Space Research Institute (Institut für Weltraumforschung, IWF) of the Austrian Academy of Sciences in Graz, and the Institute for Geophysics and Extraterrestrial Physics (IGEP) of the Technical University Braunschweig. Imperial College leads the investigation for the equatorial TC-1 satellite (PI: C. Carr) and IWF Graz leads for the polar orbiting TC-2 (PI: T. L. Zhang). Data-processing software, calibration and data correction techniques are provided by the IGEP team in collaboration with the Max-Planck Institute for extraterrestrial Physics at Garching, Germany. 
Table 2. Scientific objectives.

\begin{tabular}{|c|c|}
\hline Region and Cluster / Double Star conjunctions & FGM science objectives \\
\hline \multicolumn{2}{|l|}{ Dayside Magnetosphere 2004} \\
\hline \multirow{2}{*}{$\begin{array}{l}\text { From start of TC-1 operations in January } 2004 \text { to late spring } \\
\text { (May 2004) }\end{array}$} & Reconnection at the dayside magnetopause \\
\hline & $\begin{array}{l}\text { Relationship of low-latitude and high-latitude reconnection } \\
\text { processes. }\end{array}$ \\
\hline $\begin{array}{l}\text { TC-1 at either the bow-shock or reconnection region, with } \\
\text { Cluster } \\
\text { as an upstream solar wind monitor }\end{array}$ & Evolution and global processes of reconnection \\
\hline $\begin{array}{l}\text { Cluster and TC- } 1 \text { at either to bow-shock of the } \\
\text { magnetopause at the same time }\end{array}$ & Energy and mass transport across the magnetopause \\
\hline Cluster in the cusp with TC-1 in the reconnection region & $\begin{array}{l}\text { Formation and structure of the magnetopause. } \\
\text { Magnetopause motion in response to sheath conditions. } \\
\text { Waves and variations in boundary structure. }\end{array}$ \\
\hline \multirow[t]{4}{*}{ TC- 1 orbit skimming the magnetopause } & $\begin{array}{l}\text { Global morphology of the magnetosheath; development of } \\
\text { waves, turbulence and instabilities, the plasma depletion } \\
\text { layer as a function of local time }\end{array}$ \\
\hline & $\begin{array}{l}\text { Bow shock dynamics and sub-structure; dependency on } \\
\text { solar wind conditions. }\end{array}$ \\
\hline & $\begin{array}{l}\text { Global bow shock dynamics: Cluster, DSP and Geotail } \\
\text { observations combined }\end{array}$ \\
\hline & Hot flow anomalies \\
\hline
\end{tabular}

Nightside Magnetosphere 2004

From start of TC-2 operations in August 2004 to Autumn (October 2004)

TC-1 at the edge of the near-Earth plasma sheet and Cluster at the tail plasma sheet $\left(\sim-20 \mathrm{R}_{E}\right)$

TC-2 in the inner magnetosphere

TC-2 at the cusp
Structure and dynamics of Magnetotail current sheets

Global processes of sub-storm initiation; cause/effect of sub-storm activities in the mid-tail (Cluster) as observed at the plasma sheet edge (TC-1) and the inner magnetosphere (TC-2)

Dipolarisation during sub-storms

Sub-storm expansion signatures at the near-Earth plasma sheet

Plasma flows and Bursty Bulk-Flows

Field-aligned currents and coupling to the ionosphere

Magnetic structures in the plasma sheet; their formation and relation to sub-storm activity
The instrument consists of two tri-axial fluxgate sensors and an electronics box. The sensors are mounted on a boom, which is deployed in-orbit and extends $3 \mathrm{~m}$ away from the surface of the spacecraft. The purpose of the boom is to minimise the magnetic disturbance from the spacecraft as measured by the fluxgate sensors. The sensors are connected to the electronics by a harness which is routed along the boom to the payload platform where the electronics box is mounted. The box contains the signal-processing electronics associated with each sensor plus dual power supplies and data processing units. The instrument is therefore fully redundant and tolerant against any single component failure; a full technical description of the instrument is given in Sect. 2.

Science exploitation is extended to a large team of coinvestigators across Europe and China. The full investigator list is given in Table 1. The FGM team has interests in a wide range of scientific topics which can be addressed by combined Double Star and Cluster observations. The scientific objectives of the team are summarised in Table 2, which lists 


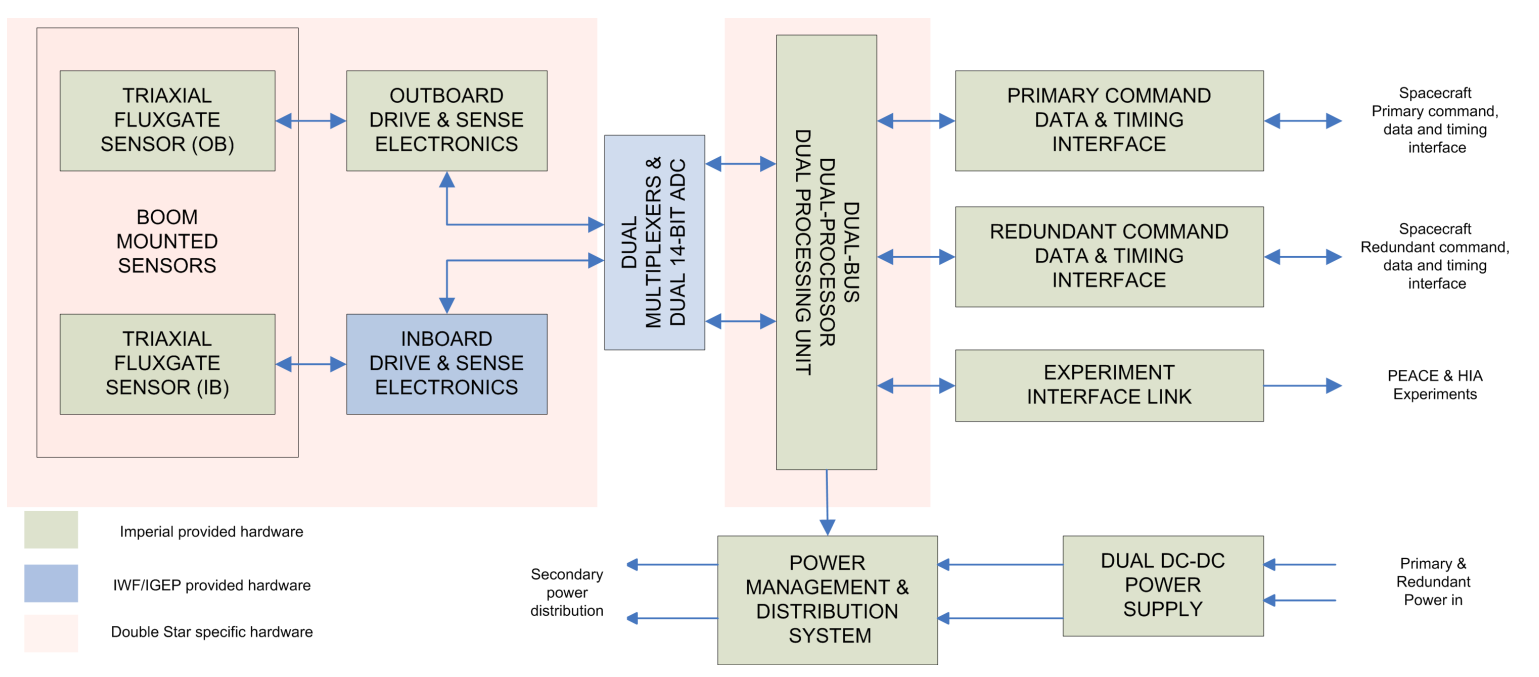

Fig. 1. Double Star magnetometer instrument block diagram.

Table 3. Instrument resources.

\begin{tabular}{ll}
\hline Outboard Sensor mass & $200 \mathrm{~g}$ (without thermal hardware) \\
Inboard Sensor mass & $230 \mathrm{~g}$ (without thermal hardware) \\
Electronics Box mass & $2630 \mathrm{~g}$ \\
Power Consumption & $3.6 \mathrm{~W}$ \\
Telemetry rate & $1211 \mathrm{bits} / \mathrm{s}$ \\
\hline
\end{tabular}

Table 4. Instrument ranges.

\begin{tabular}{lll}
\hline $\begin{array}{l}\text { Range } \\
\text { number }\end{array}$ & Range & $\begin{array}{l}\text { Digital } \\
\text { resolution }\end{array}$ \\
\hline 3 & $-128 \mathrm{nT}$ to $+127.984 \mathrm{nT}$ & $15.625 \times 10^{-3} \mathrm{nT}$ \\
4 & $-512 \mathrm{nT}$ to $+511.938 \mathrm{nT}$ & $62.5 \times 10^{-3} \mathrm{nT}$ \\
5 & $-2048 \mathrm{nT}$ to $+2047.75 \mathrm{nT}$ & $0.25 \mathrm{nT}$ \\
6 & $-8192 \mathrm{nT}$ to $+8191 \mathrm{nT}$ & $1 \mathrm{nT}$ \\
7 & $-32768 \mathrm{nT}$ to $+32764 \mathrm{nT}$ & $4 \mathrm{nT}$ \\
\hline
\end{tabular}

these objectives alongside the corresponding magnetospheric regions visited by the six spacecraft. The magnetometer instrument on Double Star TC-1 was launched and commissioned into operation in January 2004, with the TC-2 instrument becoming operational in August the same year. Double Star/Cluster has therefore visited both the dayside and nightside magnetosphere in 2004 (though for TC-2 only the latter is applicable). During the first half of 2004, TC-1 and Cluster allowed coordinated measurements to be made of the bowshock and magnetopause; these observations are outlined in Sect. 6. A preliminary study has been done of magnetic reconnection events observed at the dayside magnetopause (Pu et al., 2005). During the latter half of the year, the full complement of six spacecraft investigated the many complex

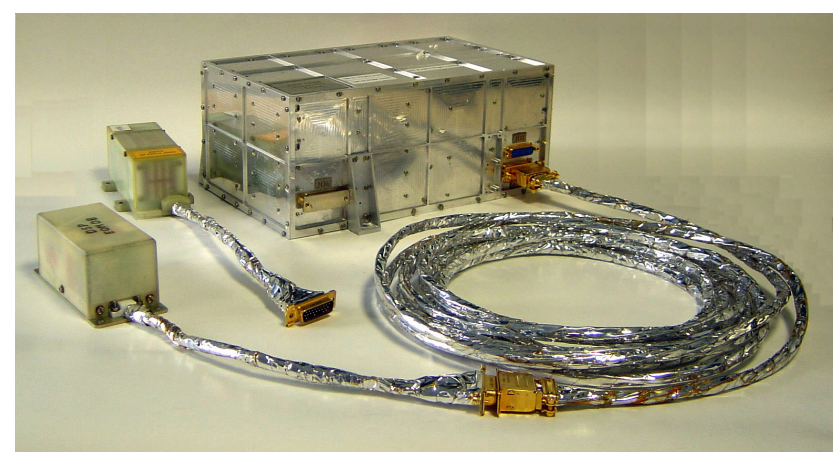

Fig. 2. Double Star magnetometer electronics box and sensors.

and inter-related phenomena of sub-storm initiation and expansion in the magnetotail. Particular focus was given to observation of dipolarisation and associated fast-flow events (Nakamura et al., 2005; Volwerk et al., 2005) and the structure of neutral sheet oscillations (Zhang et al., 2005). At the time of writing, Double Star/Cluster is starting the second visit to the dayside magnetosphere, though this is of course the first time that TC-2 has explored this region. The FGM instruments continue in good health and are operating nearly continuously; they are only switched off as required for the periodic main/redundant switching of the spacecraft sub-systems.

\section{Instrumentation}

\subsection{General description}

Both Double Star Spacecraft carry a dual sensor Fluxgate magnetometer instrument sampling the ambient magnetic field along the orbit trajectory in the frequency range DC to $10 \mathrm{~Hz}$. The FGM consists of two non-identical 
Table 5. Instrument telemetry modes.

\begin{tabular}{llll}
\hline Spacecraft TM Mode & FGM Mode & Vectors/s (Primary & Vector/s (Secondary) \\
\hline Normal Mode 1 & C & 22.45 & 3.02 \\
Normal Mode 1 & A & 12.63 & 12.63 \\
\hline
\end{tabular}

three-axis fluxgate sensors positioned at the tip, and inwards ( $0.5 \mathrm{~m}$ from tip), of a $3.1 \mathrm{~m}$ radial boom and the control electronics housed inside a box on the Spacecraft MEP (Main Equipment Platform). Both OB (Outboard) and IB (Inboard) sensors are connected to the Electronics Box via a lightweight harness. The Electronics Box houses the instrument power and sense electronics controlled by a central DPU (Data Processing Unit). The block diagram for the instrument is shown in Fig. 1 and indicates the Co-I institute responsible for each sub-unit.

The overall instrument architecture is very similar to the Cluster instruments. A dual redundant routing bus is used by the DPU to communicate with the individual instrument sub-units. While the power supply, analogue to digital converter and spacecraft interface cards are copies of the Cluster design, the Double Star instrument is significantly different from Cluster with respect to the sensors. On Cluster, both IB and OB sensors and associated electronics were provided by Goddard Space Flight Centre (GSFC). The Double Star FGM utilises two non-identical sensors and electronics. The OB sensor on both spacecraft is provided by Imperial College and the IB by IWF Graz (in cooperation with the IGEP Braunschweig). The principal reason for this arrangement was one of expediency due to the very stringent requirements with respect to the instrument delivery schedules. However it provides an interesting opportunity to make a direct comparison between two different sensor designs in similar flight conditions. Due to reasons of export control it was also necessary to redesign the instrument DPU. The newly designed Double Star sub-units are indicated by shaded colours in Fig. 1.

The instrument resources are shown in Table 3. The mass and power consumption of the instrument are moderately higher than those of Cluster. The higher mass is accounted for by an increase in outer wall thickness of the electronics box from an average value of $0.6 \mathrm{~mm}$ on Cluster to $3.2 \mathrm{~mm}$ on Double Star. The very thick box is necessary due to the increased radiation seen by the instrument in the lower orbit, and additional time spent in the radiation belts, together with a limited availability of radiation hardened electronic components due to export restrictions. The increased power is due mainly to the incorporation of two non-identical sense electronics including separate drive circuits - the power consumption of each sensor and sense electronics alone is of the order of $1 \mathrm{~W}$. A photograph of the instrument is shown in Fig. 2. The magnetometer uses five switched gain-ranges on each sensor to increase the overall instrument measurement range. Table 4 shows the dynamic range and digital resolution of the instrument for each of the five gain-ranges. These have been selected as a compromise between meeting the scientific objectives of the mission while ensuring both equatorial and polar instruments have identical builds (polarorbiting TC-2, with its lower apogee, sees a larger field compared to TC-1). The lowest range of $\pm 128 \mathrm{nT}$ is based on the pre launch orbit of TC- 1 which was not expected to regularly cross the bow shock and see the solar wind. The highest range of $\pm 32768 \mathrm{nT}$ means that for TC-2, some axes of each sensor are saturated during perigee pass. Although this causes a small increase in power consumption it does not affect the sensor performance or calibration. Magnetometer range is selected automatically in flight by the instrument software in response to a changing ambient field value.

The FGM has a reduced number of telemetry modes compared to Cluster due to the fact there is no burst mode telemetry capability on the Double Star spacecraft. Two telemetry modes currently exist and their details are shown in Table 5. As for Cluster, the data stream from each sensor is controlled by selecting one of the sensors to be primary (high data rate), the other secondary (lower data rate). Normal mode, with a resolution of 22 vectors per second from the primary sensor, was the pre-flight default operating mode of the instrument (as is the case on all four Cluster spacecraft). However, as discussed later, both instruments have operated for considerable periods in a so-called "gradiometer" mode due to spacecraft perturbations of the ambient field. This is a new mode specific to Double Star which results in equal numbers of vectors from each sensor. We use the descriptive name "gradiometer" for this mode since it is used to measure the magnetic field difference (gradient) between the outboard and the inboard sensors. This gradient is used to characterise the magnetic field interference generated on the spacecraft. Currently the default mode on TC-1 is gradiometer mode, but on TC-2 it is the normal mode with the OB sensor selected as primary.

\subsection{Sensor and magnetometer electronics}

The IB and OB sensors on Double Star provide an interesting contrast in styles. Both possess some similarities each sensor operates with conventional voltage mode detection and both use analogue electronics for the extraction of the sense signal and feedback generation. However the number of cores used, generation of the sensor drive waveform, the method of feedback, as well as the mechanical and thermal construction of the two sensors are different. 


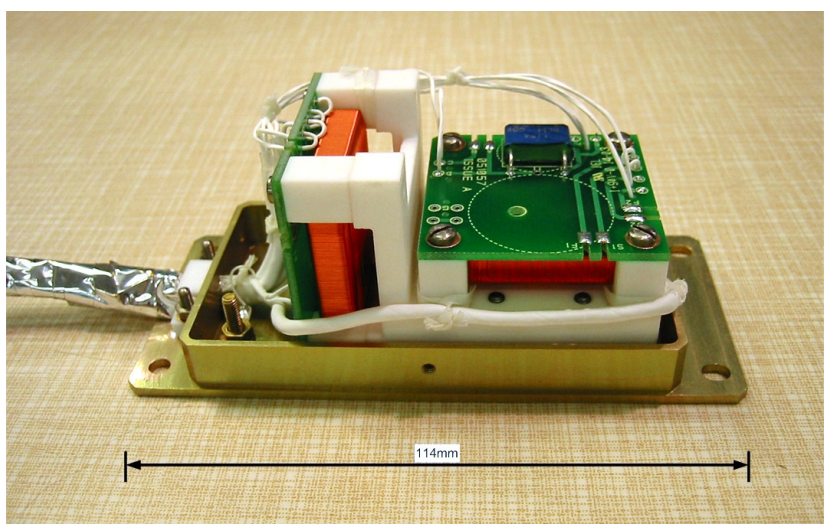

Fig. 3. OB sensor with outer cover removed showing two core implementation.

\subsubsection{Outboard Sensor and Electronics}

The outboard sensor is a lightweight construction featuring two orthogonal ring cores with dual sense and feedback windings. The ring cores are composed of a former with 22 wraps of high permeability Permalloy tape. Each ring core has a toroidally wound drive coil of 470 turns and two orthogonal square sense coils wrapped around and enclosing the ring core. The use of two sense windings per core permits the measurement of the magnetic field in two directions, thus reducing the need for a third core, in contrast to the Cluster sensor where three separate single sense wound cores are used. Further mass optimisation is achieved by combining the sense and feedback coils thereby simplifying the sensor design and reducing the total number of signal lines required in the sensor harness. In total four field measurements are performed but two of these (corresponding to a measurement from both cores in the sensor $\mathrm{x}$ direction) are averaged and are seen as a single measurement by the sense electronics.

The cores are fitted to a ceramic structure with a low coefficient of thermal expansion and attachment to the boom is achieved with an aluminium baseplate machined with an asymmetric hole pattern and small stand-off rings to allow the sensor support structure to adapt reversibly to changes in temperature while minimising changes in the sensor alignments. The boom interface is very different from Cluster, where $22 \mathrm{~mm}$ Delrin stand-offs are used to provide thermal isolation between the boom and the sensor. In contrast, the OB sensor on Double Star is thermally coupled to the boom, in order to ensure the sensor quickly follows the boom temperature such that thermal gradients across the sensor (which could lead to anomalous offset) are minimised. The sensor and its associated electronics have been designed by Ultra Electronics Ltd., and the sensor in particular bears heritage from that flown on the Cassini mission (Kellock et al., 1996). The flight sensors were manufactured by Ultra, whilst the electronics was manufactured at Imperial College. Figure 3 shows a photo of the sensor with the outer fibreglass cover removed.
A $14.98 \mathrm{kHz}$ drive and $29.96 \mathrm{kHz}$ second harmonic reference waveform are derived from an internal FGM clock. The drive frequency has been selected as a trade off between power and sensor noise. As the power resource available to FGM was quite generous, a sensor with increased power but better noise performance (compared with Cluster) could be realised. The drive is coupled into a resonant circuit via emitter-follower transistors which results in two large current spikes of alternating polarity in the drive winding once per drive cycle. Each ring core is therefore driven around its hysterisis loop deeply saturating the core material twice per drive period This so called "tank circuit" is an efficient method of ensuring deep saturation of the core material for modest power consumption (Geyger, 1965). A voltage is induced inside the sense winding in the case of non-zero external field due to the mismatching of the hysteresis loops of the half-cores. The even harmonic components of the voltage waveform are proportional to the DC magnetic field value as has been described by many authors (Primdahl, 1979; Ripka, 1992).

The sense electronics uses only the first even harmonic (which is of course the largest of the even components) to extract field information. A tuned resonant circuit filters the second harmonic (so called "sensor $2 f$ ") of the induced voltage waveform before feeding it to a preamp. The resonance allows some intrinsic gain of the small sensor $2 f$ signal the modulated inductance of the ring-core means the resonant circuit is non linear and very high sensitivities are in fact possible (Acuña, 1974). However in practise the operating point of the tuned circuit is selected so as to hold the Q value to a lower level to inhibit the effect of temperature drift on the gain. The intrinsic sensitivity of the sensor open loop is of the order $20 \mu \mathrm{V} / \mathrm{nT}$.

Following the pre-amp, the in-phase second harmonic component in the sensor voltage is measured by rectifying the signal across a capacitor via an analogue switch clocked at the sensor $2 f$ reference clock. This voltage provides the input to a differential integrator which converts the signal to a DC voltage, which is then fed back to the sense coil via a V to I converter. The three feedback voltages are directly proportional to the magnetic field components and are fed to three of the input channels of a multiplexer on the ADC card via a switched gain stage to provide for the 5 measurement ranges. It is necessary to use a parallel resonance circuit to isolate the low impedance feedback circuit from the high impedance tuned sense circuit at the second harmonic frequency. An anti-aliasing filter is included at the output stage with a $3 \mathrm{~dB}$ point of the order of $60 \mathrm{~Hz}$. The sensor includes a $24 \mathrm{~cm}$ pigtail cable which connects to the Electronics Box via a boom harness of the same construction. A simplified sketch, showing the electronics of a single axis magnetometer, is shown in Fig. 4.

\subsubsection{Inboard sensor and electronics}

The inboard sensor (see Fig. 5) is a (refurbished) spare model from the Equator-S mission (Fornacon et al., 1999). 


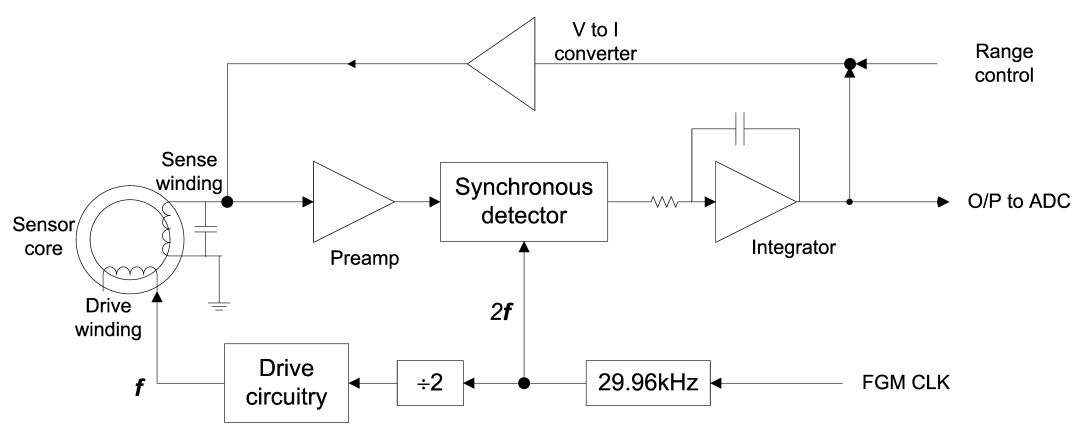

Fig. 4. Block diagram of OB sensor detection electronics (single axis).

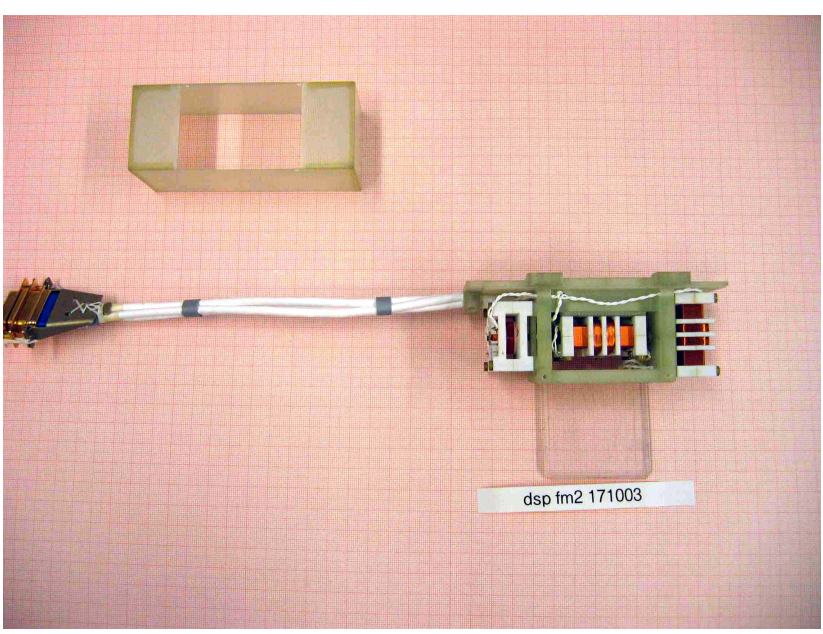

Fig. 5. IB sensor triad with pigtail cable/connector and opened protection housing.

The sensor system contains three identical single-axis fluxgate sensor elements mounted perpendicularly to each other. The soft magnetic ringcore, a 6-81 moly-permalloy tape with $2 \mathrm{~mm}$ width and $20 \mu \mathrm{m}$ thickness placed in a nonferromagnetic NiMo bobbin with a diameter of $13 \mathrm{~mm}$, is positioned in the middle of a support bobbin made from Macor ceramic. Each ceramic bobbin carries two separate windings: one for feedback and the second for sense. The (protective) sensor housing is manufactured of epoxy material and includes small $(<2 \mathrm{~mm})$ stand-offs to isolate the sensor from the boom interface plate. The sensitivity of the sense coil is approximately $15 \mu \mathrm{V} / \mathrm{nT}$.

The sense circuit contains an instrumentation input amplifier with a passive band-pass network tuned to the second harmonics of the drive frequency, two passive notch filters for a further suppression of the first and third harmonic of the drive frequency and a circuit (analogue switch plus passive low pass filter) for the rectification of the field proportional $2 f$ signal. The IB drive frequency is $9.767 \mathrm{kHz}$.

The feedback circuit enables voltage feedback for the two lower measurement ranges and current feedback for the three

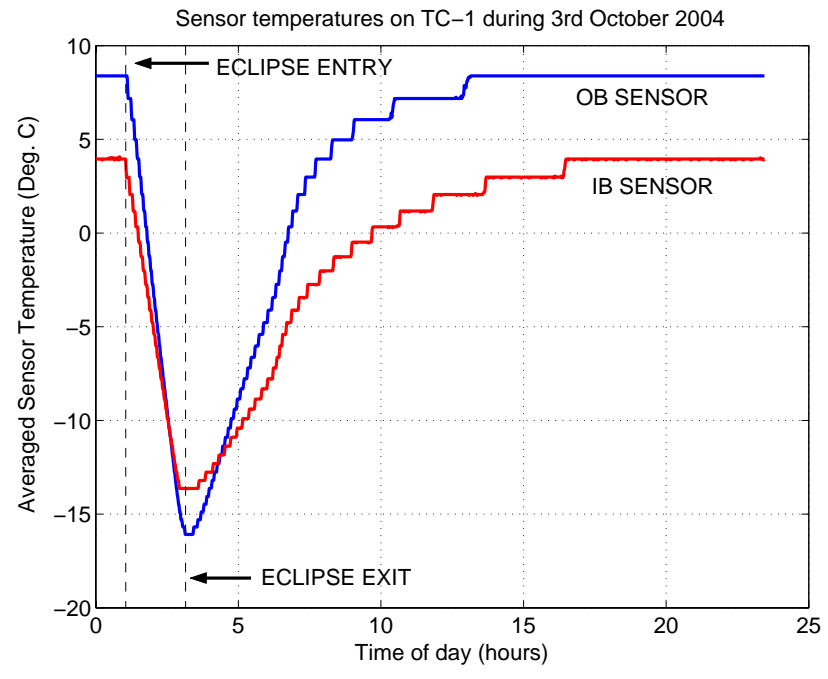

Fig. 6. TC-1 sensor temperatures through earth eclipse passage.

higher ranges. The feedback signal, in general, is driven from the amplified output voltage of the low pass filter mentioned above (i.e. no integral regulation) which allows an operation of the sense electronics without feedback (the feedback connections can be opened via latching relays) but with the drawback of an increased scale factor drift of the more sensitive ranges.

\subsection{Thermal protection}

Both OB and IB sensors and pigtails are wrapped in an multilayer insulating (MLI) blanket composed of a 2-mil outer layer of VDA Kapton with an ITO coating for good surface conductivity and interleaved layers of perforated Mylar and Dacron spacer. The MLI wraps around the sensor and boom in the region of the sensor and has been manufactured by Austrian Aerospace. Although the sensors have different thermal interfaces neither sensor possesses a heater - the combination of MLI and reasonably short eclipses ensures the sensor cores do not see significant thermal cycling and in-flight temperatures are well within both sensors' operational regimes. A plot of the measured temperature profile for a TC-1 orbit is shown in Fig. 6, demonstrating that the 
OB sensor which is thermally coupled to the boom responds to temperature changes much faster than the IB sensor. The sensor temperatures are monitored by non-magnetic thermistors inside the sensor housing.

\subsection{Digital electronics and software}

The Double Star DPU uses a Dynex MAS31750 processor implementing the MIL-STD-1750A instruction set together with 8 kwords of PROM and 16 kwords of RAM. An Actel RT1460A FPGA provides the glue logic for the board as well as the control registers for accessing the instrument sub-units over the routing bus. The FPGA and RAM are different to Cluster and the TID (total ionising dose) tolerance of the FPGA is of the order $20 \mathrm{kRad}$, which is much lower than the equivalent radiation hardened part on Cluster, hence the requirement for additional shielding provided by the external box.

The FGM uses a 16-bit Crystal CS5016 Analogue to Digital Converter (ADC) together with a 16-channel multiplexer controlled by the DPU. Ten channels are used in total (six field components and four housekeeping channels). The ADC sub-unit is provided by IWF Graz and is identical to the design used for the Cluster magnetometers. Primary sensor magnetic field values are sampled by the ADC in the order $\mathrm{x}$, $y, z$ a rate of 201.969 vectors per second. The internal $2^{23} \mathrm{~Hz}$ clock is divided down to $240 \mathrm{kHz}$ and used to ensure sampling of the vector occurs at precisely regular intervals (better than $1 \mu \mathrm{s}$ accuracy). The first vector of each telemetry frame is time stamped on board using the spacecraft provided high frequency clock $(4096 \mathrm{~Hz})$ which is frozen and read by the DPU at the instant of the $y$ acquisition. The time between consecutive vectors is $4.95 \mathrm{~ms}$ and the time between vector components is $173 \mu \mathrm{s}$. A Gaussian digital filter is used to match the rate and bandwidth of the transmitted vectors to the available telemetry rate.

The timing of the raw secondary vector sampling is not as carefully controlled as that of the primary and the time between vector components is of the order $4.9 \mathrm{~ms}$. The secondary data is unfiltered and essentially the spot value of the magnetic field is transmitted to ground. The poor time resolution of the secondary data compared to primary should not be an issue as usually only the filtered higher rate primary data makes up the usable scientific product. However the presence of $\mathrm{AC}$ magnetic perturbations from the solar array on Double Star means that, on TC-1 in particular, the secondary data stream has increased importance compared to Cluster. A "gradiometer" style telemetry mode with equal number of vectors from both OB and IB sensors has been implemented as a patch to the DPU operating software and is now incorporated into the instrument "power-on" sequence. This has been achieved by increasing the sample rate of the secondary data and increasing the decimation of the primary data. It is necessary to disable the filtering of the primary data by tele-command prior to entering this mode.

\section{Instrument performance}

The instrument performance was analysed on the ground at the IWF institute in Graz. This facility includes a temperature controlled low field mu-metal can, allowing sensor performance criteria to be measured across a temperature range chosen to exceed the temperatures predicted for flight operations $\left(-35^{\circ} \mathrm{C}\right.$ to $\left.+25^{\circ} \mathrm{C}\right)$. The measurements were taken for each measurement range. The results are summarised in Table 6.

As can be seen in Fig. 6, the actual variation in sensor temperature over a typical TC-1 orbit, including an eclipse of greater than $2.5 \mathrm{~h}$, is of the order of $30^{\circ} \mathrm{C}$ : from a minimum of around $-17^{\circ} \mathrm{C}$ to a maximum temperature of $12^{\circ} \mathrm{C}$.

\subsection{Sensor noise}

The sensor noise density was measured for each axis in each range, at temperatures of $30^{\circ} \mathrm{C}, 10^{\circ} \mathrm{C}$ and $-35^{\circ} \mathrm{C}$. The feedback voltage for each axis, with appropriate range gain applied, is routed to a separate connector to allow it to be analysed using a spectrum analyser, measuring noise density of the sensor's analogue signal output. This is scaled to a measurement in $\mathrm{pT} / \sqrt{ } \mathrm{Hz}$. The results presented are those measured at $1 \mathrm{~Hz}$.

The OB sensor noise as measured at the analogue signal output of the Double Star flight models is less than $5 \mathrm{pT} / \sqrt{ } \mathrm{Hz}$ at $1 \mathrm{~Hz}$ across the whole temperature range. This figure compares very favourably with other space-borne fluxgates and translates to a RMS analogue noise level of $11 \mathrm{pT}$ in the bandwidth 0.1 to $10 \mathrm{~Hz}$. This noise level is of the order of the digital resolution of the magnetometer of $16 \mathrm{pT}$ in the most sensitive range (range 3 ). The results for flight model 1 , flown on the TC-1 spacecraft, are shown in Fig. 7.

The Double Star digital electronics design is based on Cluster design heritage. A further iteration of the design, between the Double Star Engineering-Qualification Model (EQM) and Flight Model (FM), produced a significant improvement in noise performance. The Cluster magnetometer (and also the Double Star EQM), uses a $8.39 \mathrm{MHz}$ (i.e. $2^{23} \mathrm{~Hz}$ ) crystal oscillator as master clock for both sensor drive and timing of the ADC sampling by the DPU. In the Double Star flight model design, these two functions are separated; the sensor drive uses an $8.39 \mathrm{MHz}$ clock whilst the ADC sampling is timed from a separate $8 \mathrm{MHz}$ clock. This considerably reduces the noise induced in the sensor by jitter on the clock line introduced by the DPU accessing the ADC. The noise density of the qualification model of approximately $19 \mathrm{pT} / \sqrt{ } \mathrm{Hz}$ at $1 \mathrm{~Hz}$ was reduced to less than $5 \mathrm{pT} / \sqrt{ } \mathrm{Hz}$ for the flight models due to this modification.

The IB sensor analogue noise density of the Double Star flight models is below $13 \mathrm{pT} / \sqrt{ } \mathrm{Hz}$ at $1 \mathrm{~Hz}$ at all temperatures above $-35^{\circ} \mathrm{C}$. 
Table 6. Magnetometer instrument performance criteria summary.

\begin{tabular}{lll}
\hline Performance Criteria & Double Star OB flight sensor & Double Star IB flight sensor \\
\hline $\begin{array}{l}\text { Analogue Voltage Noise density @ } 1 \mathrm{~Hz} \text { for } \\
\text { temperature range }-35^{\circ} \mathrm{C} \text { to } 25^{\circ} \mathrm{C}\end{array}$ & $<5 \mathrm{pT} / \sqrt{\mathrm{Hz}}$ & $<13 \mathrm{pT} / \sqrt{\mathrm{Hz}}$ \\
\hline Offset Drift between $-35^{\circ} \mathrm{C}$ and $25^{\circ} \mathrm{C}$ & $<0.03 \mathrm{nT} /{ }^{\circ} \mathrm{C}$ & $<0.07 \mathrm{nT} /{ }^{\circ} \mathrm{C}$ \\
\hline Scale Factor Drift between $-35^{\circ} \mathrm{C}$ and $25^{\circ} \mathrm{C}$ & $<0.05 \%$ & $<0.05 \% \mathrm{R} 5-\mathrm{R} 7$ \\
& & $<0.4 \% \mathrm{R} 4$ \\
& & $<1.5 \% \mathrm{R} 3$ \\
\hline
\end{tabular}

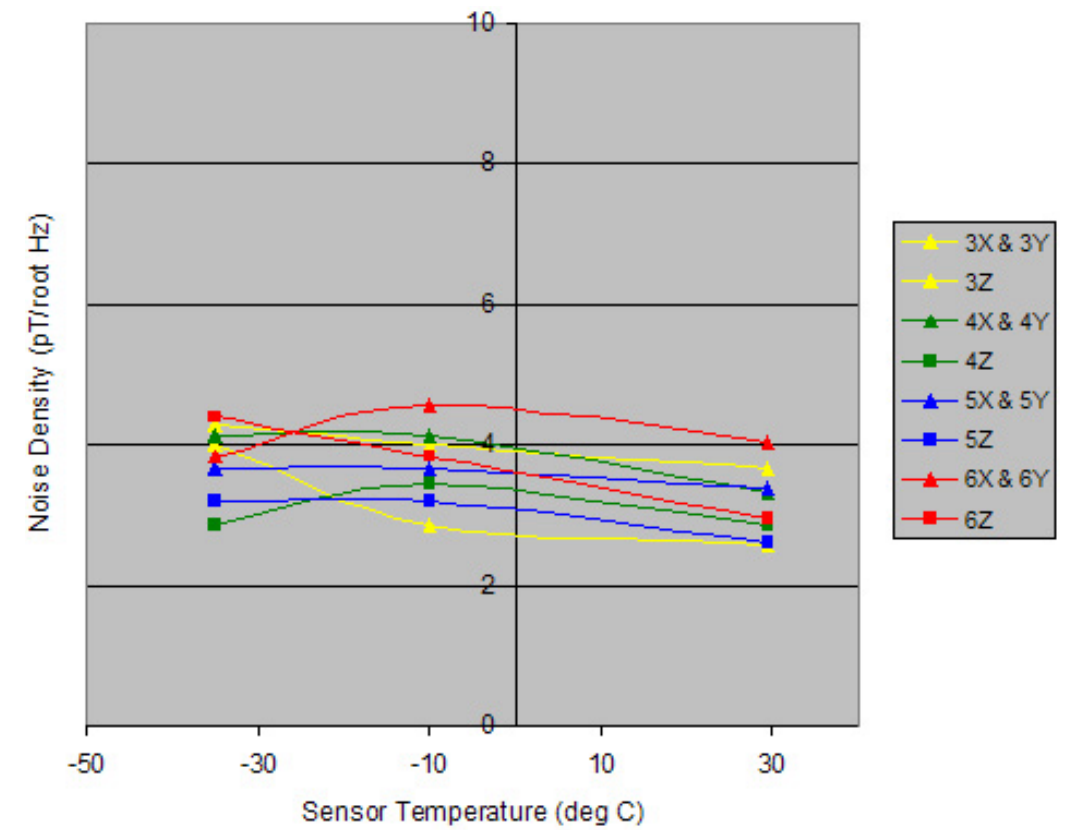

Fig. 7. Double Star flight model 1, OB sensor noise density temperature stability.

\subsection{Sensor offsets}

Sensor offset stability is of critical importance to the realisation of the science objectives. The offsets for the $\mathrm{x}$ axes of the flight model 1 sensors in range 3 are shown in Fig. 8. The error bars on the graphs indicate the uncertainties in the measurements, which are most pronounced in range 3 , the most sensitive range. The drift in sensor offsets was measured for the sensors in the mu-metal can for the same temperature range. For the Double Star flight OB sensors, the offset drift was below $0.03 \mathrm{nT} /{ }^{\circ} \mathrm{C}$ on all axes, in all ranges across the temperature range. For the Double Star flight IB sensors, the offset drift was below $0.07 \mathrm{nT} /{ }^{\circ} \mathrm{C}$ on all axes in all ranges across the temperature range.

\subsection{Sensor linearity}

The Double Star instrument has stringent linearity specifications across the full scale of each range, and for the drift of that linearity with temperature. The scale factor is not routinely determined in flight as discussed in Sect. 5, so ground measurements are particularly important. The linearity across full scale was measured for the FM Double Star OB sensor as 1 part in 10000 . The drift of this scale factor across the temperature range measured is less than $0.05 \%$ on all axes in all ranges.

For the IB sensors, the linearity across full scale was measured to be 1 part in 10000 , as for the OB sensor. The drift with temperature for the higher ranges 5, 6 and 7 is $0.05 \%$ while for the more sensitive ranges 4 and 3 the drift is less than $0.4 \%$ and $1.5 \%$, respectively.

\section{Data processing: summary of data throughput and data products}

The FGM data processing software is composed of a number of distinct functional units that can be combined in a number of different ways to allow the maximum flexibility, however 

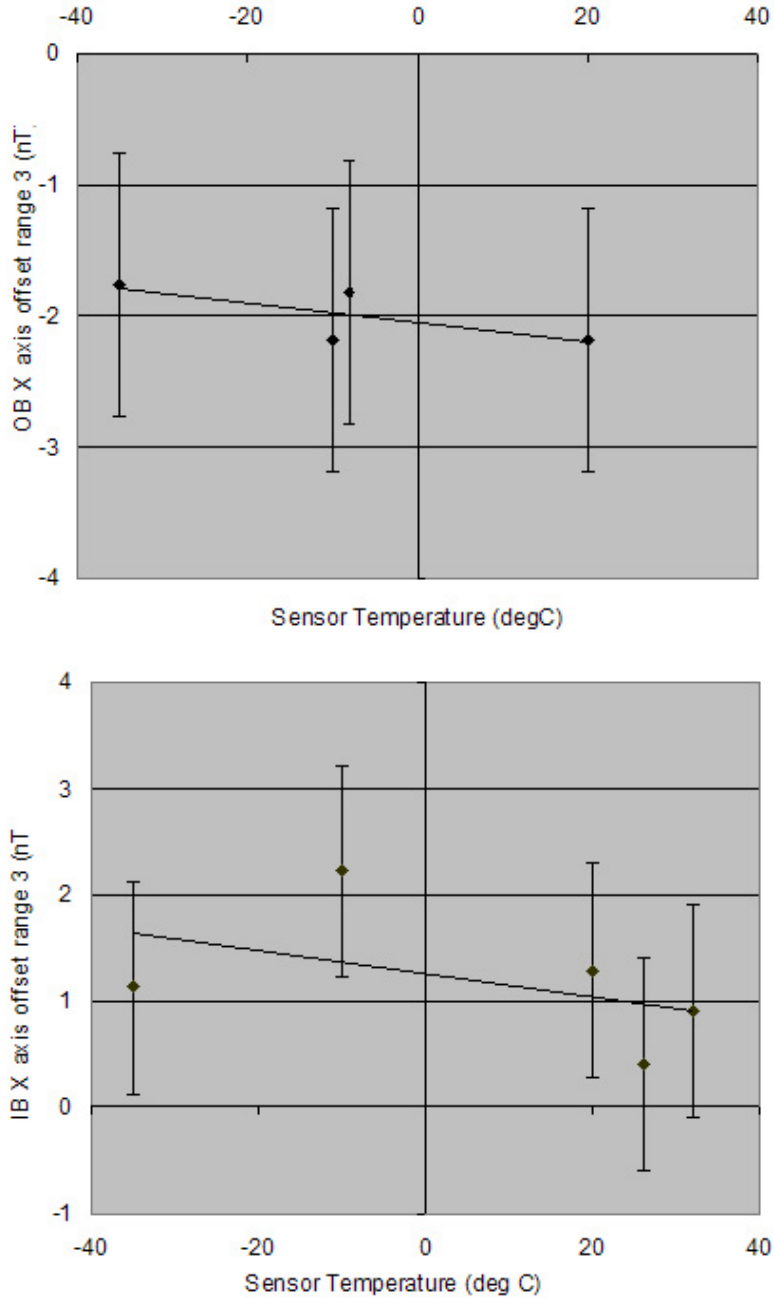

Fig. 8. Sensor Offset Temperature stability for FM1 OB and IB Sensors, $\mathrm{x}$ axes, range 3 .

generally a limited number of configurations are used. The most common arrangement is illustrated by Fig. 9 .

The primary tasks of the data processing software are

1. the conversion of the raw, compressed telemetry into a format more suitable for further processing;

2. reconstruction of the measurement time of each vector;

3. the recovery, as near as is practical, of the actual ambient field present at the location of the spacecraft by correcting for the spacecraft field and instrumentation influences;

4. transformation from the spinning sensor coordinate system into standard geophysical coordinate systems.

Data is passed between the different processing elements using a standard structure. Conversion into this format is achieved using the module fgmtel.c. This module's primary function is to unpack the data into floating point numbers, applying the appropriate scaling factors for each range, to generate an un-calibrated vector in the sensor coordinate system. The output of this module is in units of nT. The appropriate time and spin phase for each vector is also derived at this stage, and added to the data structure. Additionally fgmtel. $c$ can be used to select between the two sensors. The second stage of processing is the application of the calibration variables to the data using the program module fgmcal.c. Calibration values are generated externally to the processing chain and consist of 12 values for each combination of the two sensors, two analogue to digital converters and five ranges, a total of 240 distinct values. The magnetic field vectors produced at this point are still in a sensor related coordinate system, but are orthogonal and represent an accurate value. The next module, fgmhrt.c, is used to convert the data from the sensor coordinate system called FGM Spin Reference (FSR) into various standard coordinate systems. Commonly used systems are the Geocentric Solar Ecliptic system (GSE), the Geocentric Solar Magnetospheric system (GSM), Geocentric Equatorial Inertial system (GEI), and Spacecraft-Sun system (SCS). This conversion includes "de-spinning", or removing the sinusoidal modulation of the field caused by the spacecraft's rotation. The function of fgmhrt.c can be considered as the application of two transformation matrices, $\mathbf{c}^{(a t t)^{-1}}$ and $\mathbf{c}^{(\text {spin })^{-1}}$, the former matrix representing the conversion to GSE from the de-spun reference system, and the second matrix representing a rotation matrix relating to the spin angle of the sensors at the time the field vector was sampled.

$\boldsymbol{B}_{\boldsymbol{G S E}}=\mathbf{c}^{(a t t)^{-1}} \mathbf{c}^{(\text {spin })^{-1}} \boldsymbol{B}_{\boldsymbol{F} S \boldsymbol{R}}$

A further stage, fgmav.c, is often used after this point to average the data over a period of time, or a revolution of the spacecraft, depending on the usage of the data. If fgmav. C is omitted, data is produced at the highest data rate for the sensor at this time, which is around 22 vectors/s for the primary sensor when the instrument is operating in "normal" mode.

A final processing stage, fgmvec.c, is used to convert the standard data structure used within the processing chain into tabulated ASCII data, typically containing each vectors time and the three components of the magnetic field.

Other processing functions exist to (i) filter specific time intervals; (ii) filter specific instrument ranges for calibration; (iii) pre-process auxiliary data file such as spacecraft attitude, orbit, and event information; (iv) produce specially formatted output data, and (v) aid in debugging.

The raw FGM data is received by the spacecraft ground stations, forwarded to the DOMC (Double Star Operations and Management Centre) at CSSAR (Centre for Space Science and Applied Research, Chinese Academy of Sciences), and in turn forwarded on to the EDDS (European Data Disposition System) at IWF Graz. FGM operations, at Imperial College London, in turn receives a copy of all the relevant files using the open source utility RSYNC, and a secure encrypted link over the Internet. IWF routinely produces spin and one minute average data for the SPDB and PPDB (Summary Parameter and Prime Parameter Data Base), essentially 


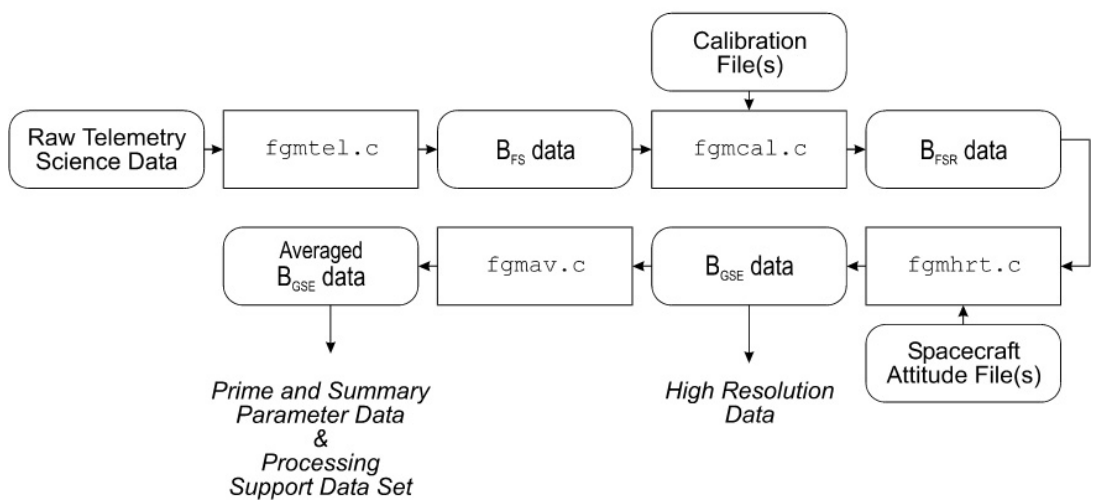

Fig. 9. Simplified data flow diagram of the FGM data processing software.

using the processing chain described above, the SPDB being averaged over one minute, and PPDB over a single spin. Data is also used at spin average by other Double Star instruments, to support the production of their data sets, this is termed the Processing Support Data Set (PSDS).

Additionally for TC-1, due to the significant error introduced into the magnetic field measurements caused by large (unintentional) spacecraft fields, additional processing steps are needed to produce usable data of publication quality. For TC-1, operation is generally in gradiometer mode, so equal numbers of vectors are returned from both the primary (outboard) and secondary (inboard) sensors. A more detailed justification and explanation of the cleaning process is given in Sect. 5.2. Functionally, the normal data processing chain is used twice, once for each of the sensors. The difference between the two data sets produced is a measure of the disturbing field from the spacecraft. This can then be used to remove a significant proportion of this interfering signal from the data for one sensor (normally the outboard sensor).

\section{In-flight calibration}

\subsection{Calibration parameters}

The accurate in-flight determination of calibration parameters is of vital importance to achieve the desired accuracy from the magnetic field experiment on each spacecraft. This is particularly the case when magnetic field measurements are used together from both spacecraft and when combining these with measurements from other missions such as Cluster.

The full calibration process involves converting the output from the magnetometer, which is received via telemetry and is in binary units, into physical units of nT in a standard orthogonal coordinate system. This process is represented by the instrument model. The instrument model contains the calibration parameters which are to be determined in-flight and in addition, parameters which were determined on the ground before launch and parameters which are determined by other means. The full details of this instrument model and the parameters used to define it was described by Balogh et al. (2001). The shared heritage between the Double Star and Cluster FGM means that the instrument models are essentially the same.

The components of the instrument model which are relevant for in-flight calibration comprise a $3 \times 3$ matrix and a 3 component vector. Each component of the $3 \times 3$ matrix is an expression which contains sensor gains and angles. There are two different angles associated with each sensor and these are used to define the magnetic orientation of the sensors relative to the spacecraft. This matrix scales and transforms the magnetic field measurements into $\mathrm{nT}$ in an orthogonlised coordinate system. The three component vector is the set of sensor offsets which, along with each component of the $3 \times 3$ matrix, are instrument range-dependent. From this description of the calibration transformation it can be seen that there are 12 calibration parameters which need to be determined and in addition to this there is the instrument range dependence to take into consideration. There are 3 gains, 3 offsets and 6 angles which cover the three sensor components. Fourier analysis of in-flight magnetic field data allows the determination of 8 of these 12 calibration parameters for each instrument range. The remaining parameters must then be estimated by other means which includes the use of ground calibration, data either side of instrument range changes and if it is available, the use of solar wind data.

\subsection{TC-1}

During the commissioning of TC-1 it was determined from the initial calibration efforts that there was significant spacecraft interference superimposed on the space environment magnetic field. This interference takes the form of a spin period signal which can be seen in the high time resolution data of both the inboard and outboard magnetometer data. The amplitude of the signal is larger at the inboard sensor which is expected to be the case for an interference signal which originates from the spacecraft. On further investigation it has been found that the source of this interference is the spacecraft current distribution and shunting system associated with the solar generators. 

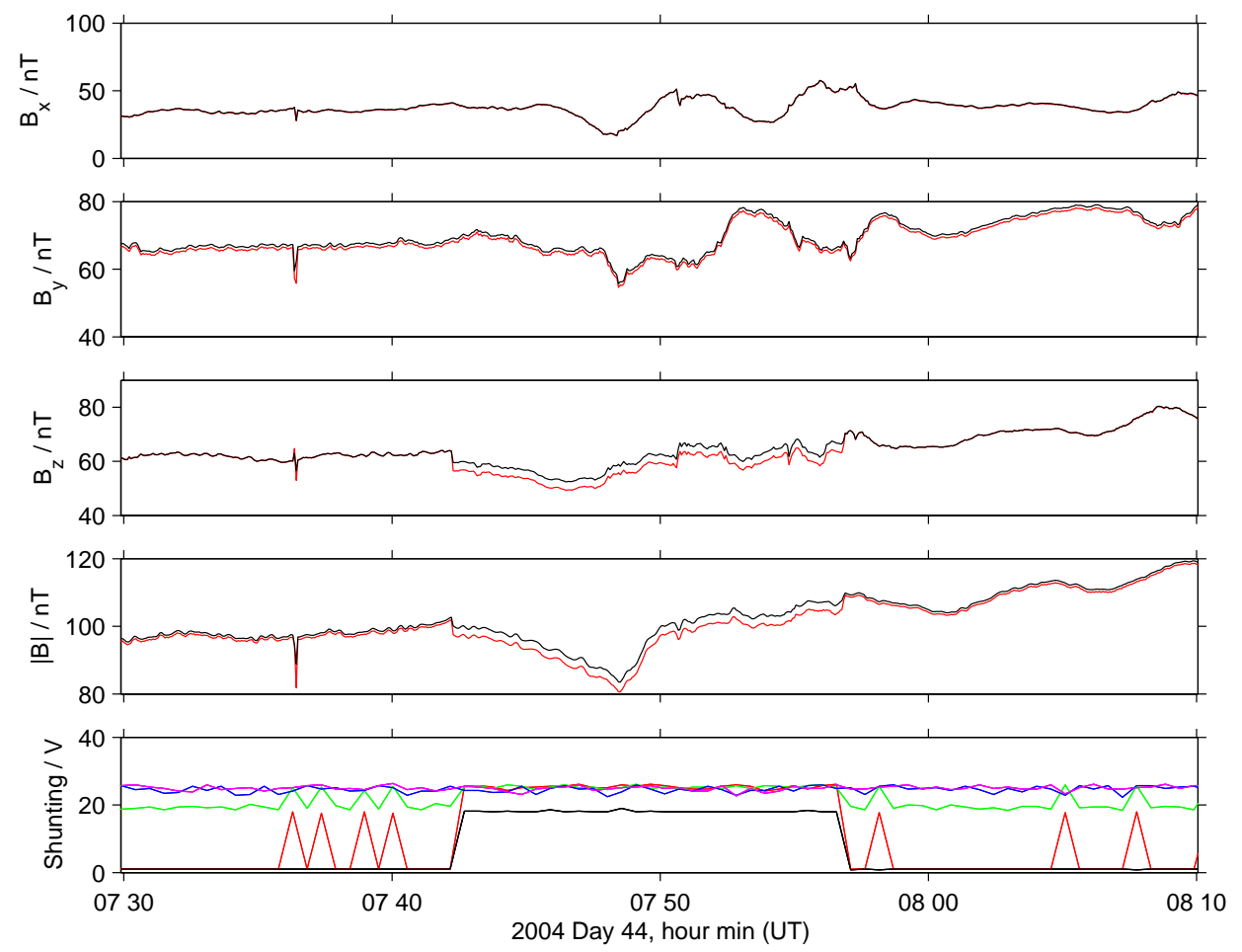

Fig. 10. A comparison of discontinuities seen in the magnetic field measured at both the inboard and outboard sensors with changes in the spacecraft solar generator shunting levels. The top three panels show the inboard (red) and outboard (black) magnetic field in the GSE x, $\mathrm{y}$, and $\mathrm{z}$ directions respectively. The fourth panel shows the magnetic field magnitude again for the inboard and outboard sensors. The fifth panel shows the shunting voltage for shunting level 1 (black), level 2 red), level 3 (green), level 4 (blue) and level 5 (magenta).

The spacecraft interference signal originating from the solar power system has proved to be a major set back for the inflight calibration of the magnetometer experiment on TC-1. The reason for this is associated with the properties of the interference signal. When the magnetic field data is de-spun and a Fourier transform taken, it is clear that the interference signal introduces significant spin harmonics into the data. The analysis which is central to in-flight calibration uses the spin harmonics present in the de-spun magnetic field data to estimate many of the calibration parameters. However, it is not possible to determine what contribution of the spin harmonics comes from the spacecraft interference, and how much is due to corrections that need to be made to calibration parameters. In addition to these complications involved with determining calibration parameters, the actual process of removing the spacecraft interference magnetic field from high time resolution data is a significant problem and one which has yet to be solved. In order to make progress at this stage, efforts have been concentrated on a method for calibrating and removing the spacecraft field from spin-averaged data.

De-spinning and then averaging the magnetic field data over precisely the spin of the spacecraft considerably reduces the interference problem. In the two spin-plane de-spun sensor components, averaging the magnetic field over a spin cancels out a major part of the interference, because the interference in de-spun coordinates is made up of sinusoidal components which have frequencies at multiples of the spacecraft spin frequency. The component of the interference that is left is due to asymmetries in the spacecraft generated field, and appears as a constant offset on each spin averaged data point of the two spin plane components. The effect of spin averaging on the de-spun spin axis magnetic field component is to reduce the interference again to a constant offset but in this case no cancelling of the field occurs. A complication to this situation is that the offsets to each of the magnetic field components only remains at a particular level while the spacecraft is operating in one current shunting configuration. In general the spacecraft changes the current shunting mode several times a day as the spacecraft power demand changes. This results in discontinuities in the spin averaged magnetic field data as the offset due to the spacecraft interference changes throughout the day. Figure 10 shows how the changes in the spacecraft shunting configuration result in discontinuous changes in the measured magnetic field.

Before a method can be applied to remove the spacecraft interference from the spin averaged data, an initial calibration estimate must be applied to it. In order to determine these initial calibration parameters, periods of data are required where there is no spacecraft interference. This data can be found when TC-1 is in solar eclipse regions of the orbit, and is powered by batteries rather than the solar generator. Only a rough estimation of some calibration parameters 
can be achieved using eclipse data, as it is necessary to apply this calibration to data which can be far from the point where the calibration analysis is performed. The reason for this is that during the course of the year the eclipsed region drifts gradually through all the different magnetospheric regions encountered throughout the spacecraft orbit. As different regions are encountered, the instrument range tends to change along with the associated calibration parameters. The eclipse calibration is the first step in producing the spin averaged data product.

Having applied the eclipse calibration and spin averaging to the data, a method for removing the offset on each sensor component due to the spacecraft interference is required. The total magnetic field measured by the fluxgate magnetometer can be considered as the sum of the spacecraft interference field and the ambient magnetic field. The intention is to estimate the interference magnetic field at the outboard sensor and therefore allow the extraction of the ambient magnetic field which is of interest. The key to this method lies in the fact that the interference field has its source at the spacecraft so the rate at which the field is falling off can be detected and measured by comparing measurements from the inboard and outboard sensors. The ambient magnetic field on the other hand is uniform on the scale of the distance between the two sensors which means the same field measurement is expected to be made at both the inboard and outboard sensors. The use of the inboard and outboard magnetic field measurements to distinguish between the ambient field and fields which have their source at the spacecraft is known as the dual magnetometer method (Ness, 1971).

In the case of TC-1, the expression for calculating the spacecraft interference contribution to the magnetic field measured at the outboard sensor, is derived by first approximating the interference to a dipole field. The quantity that is required at this stage is $B_{O S}$ which is the magnetic field due to the spacecraft interference as measured at the outboard sensor. The magnetic field quantities that are actually measured are $B_{o t}$ and $B_{i t}$ which are the total magnetic field at the outboard and inboard sensors respectively. The total magnetic field consists of the interference plus the ambient field (subscript $a$ ). As a simplified demonstration here, it is assumed that the magnetic dipole moment is aligned with the spacecraft spin axis direction, and is located at the centre of the spacecraft. This means that the magnetic dipole field as measured in the spin axis sensor direction is given by $\frac{m\left(3 \cos ^{2} \theta-1\right)}{4 \pi \mu_{0} x^{3}}$ where $m$ is the magnitude of the dipole moment, $\theta$ is the angle between the direction of the dipole moment and the spin axis sensor direction (zero in this case), $\mu_{0}$ is the permeability of free space and $x$ is the distance between the source of the dipole field and the sensor. Substituting this dipole field expression into the magnetic field ratio in Eq. (2) shows that this ratio of the interference magnetic field at the outboard sensor to the difference between the total field measured at the inboard sensor and outboard sensor is a constant, $k_{o}$. It is also true in the general case of a dipole orientated in an arbitrary way to the sensor directions that the ratio $\frac{B_{o s}}{B_{i t}-B_{o t}}$ for each sensor component is equal to a constant. However the constant for each sensor component does not necessarily take the same value.

$$
\begin{gathered}
\frac{B_{o s}}{B_{i t}-B_{o t}}=\frac{B_{o s}}{B_{i s}+B_{i a}-B_{o s}-B_{o a}}=\frac{B_{o s}}{B_{i s}-B_{o s}} \\
\frac{\left(\frac{m\left(3 \cos ^{2} \theta-1\right)}{4 \pi \mu_{0} x_{o}^{3}}\right)}{\left(\frac{m\left(3 \cos ^{2} \theta-1\right)}{4 \pi \mu_{0} x_{i}^{3}}\right)-\left(\frac{m\left(3 \cos ^{2} \theta-1\right)}{4 \pi \mu_{0} x_{o}^{3}}\right)}=\frac{1}{\left(\frac{x_{o}}{x_{i}}\right)^{3}-1}=k_{o}
\end{gathered}
$$

Rearranging Eq. (2) into the form $B_{o s}=k_{o}\left(B_{i t}-B_{o t}\right)$ gives the expression which is used to calculate the spacecraft interference at the outboard sensor for each sensor component. In order to use this expression, values for the three constants, one for each sensor direction, must be determined. As previously discussed, changes in the spacecraft power shunting level, are accompanied by changes in the spacecraft interference field and this is seen as a discontinuous field change in the magnetic field data. The size of these discontinuous changes can be used to determine the constants $k_{o}$ for each sensor component. These constants are determined empirically rather than using Eq. (2) because the real spacecraft interference is not likely to be exactly described by a single dipole and the orientation of the interference is unknown. Equation (3) is used to calculate a distribution of $k_{o}$ values from the data by looking at many shunting level changes and a statistical analysis of these results gives an empirical value for $k_{o}$ that can then be used to calculate $B_{o s}$. It is thought that shunting between different levels will lead to different values for the constant $k_{o}$ and this must be taken into consideration when doing the statistical analysis. Exact values for these constants have not been calculated yet.

$$
k_{o}=\frac{B_{o t 1}-B_{o t 2}}{\left(B_{i t 1}-B_{o t 1}\right)-\left(B_{i t 2}-B_{o t 2}\right)}
$$

The magnetic field experiment on TC-1 was originally designed to provide magnetic field measurements at a higher data rate at the outboard, primary sensor than at the inboard secondary sensor, as described in Sect. 3.4 of this paper a new telemetry mode called the gradiometer mode has been defined and the onboard software patched. When operating in gradiometer mode, the data rate is the same from the inboard and outboard sensors, which is essential in order to obtain the most accurate results from the spacecraft interference calculations described above. Due to fluctuations in the difference between inboard and outboard magnetic field from one spin averaged data point to the next, in practice it is the median of $B_{i t}-B_{o t}$ which is used in the calculation of $B_{o s}$. The median is calculated over periods of time when the spacecraft is in one particular shunting mode. Having calculated $B_{o s}$ using the median of $B_{i t}-B_{o t}$ it is a simple matter to calculate the actual magnetic field measured by the outboard sensor with the interference field removed by using $B_{o a}=B_{o t}-B_{o s}$. Figure 11 shows a period of time when the spacecraft shunting mode switches twice. The discontinuous changes in both the 

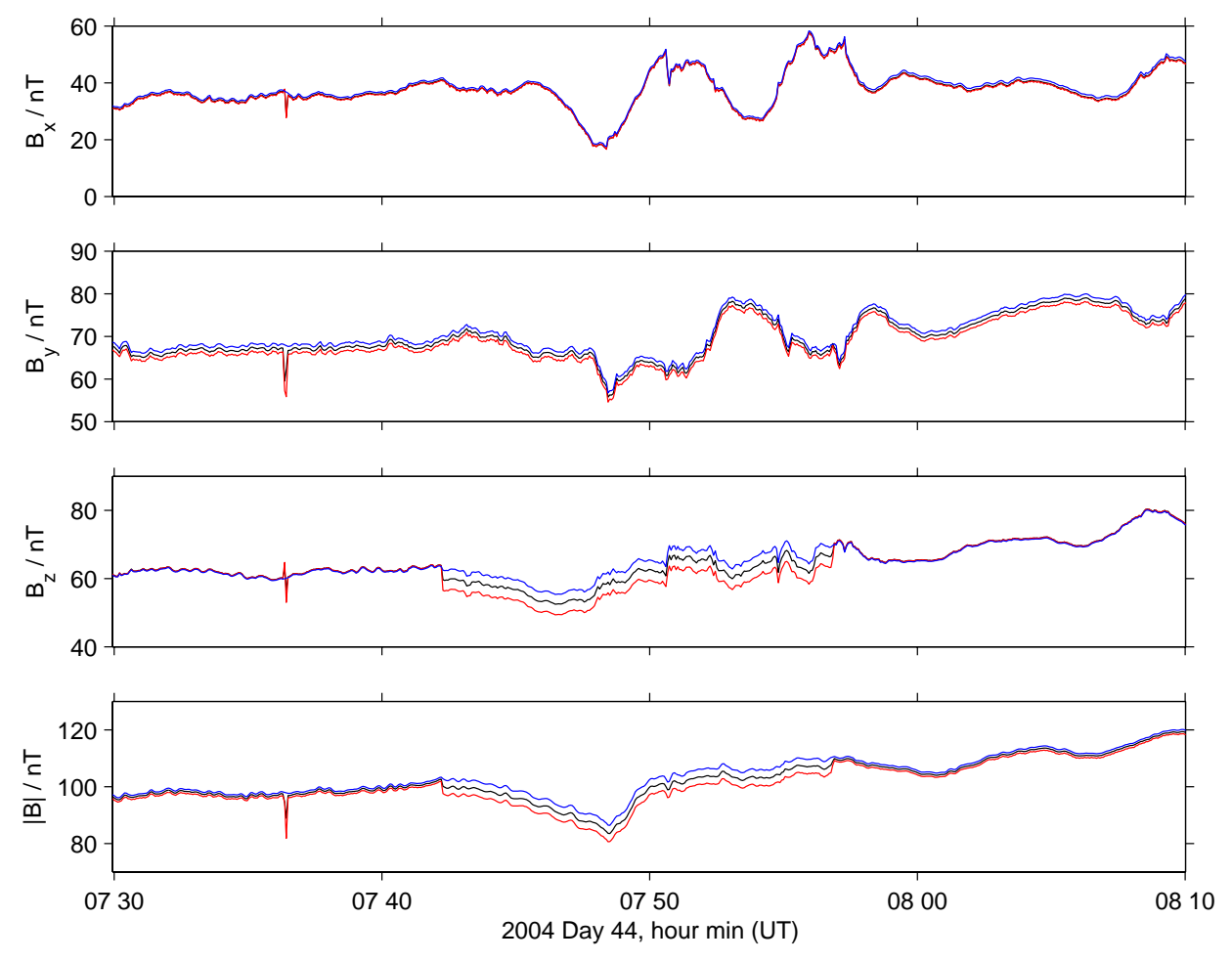

Fig. 11. A comparison between the inboard (red) and outboard (black) magnetic field before removing the spacecraft interference with the outboard magnetic field after the interference removal shown in blue. The top three panels are the GSE x, y and z magnetic field components respectively and the fourth panel is the magnetic field magnitude.

inboard and outboard magnetic field can clearly be seen, and as expected it is the spin axis field in the GSE $z$ direction that is effected most by the spacecraft interference. The blue line in Fig. 11 is the magnetic field with the spacecraft field removed and even with an approximate $k_{o}$ used in this case, the discontinuous field changes at the shunting level shifts are almost completely removed.

When the spacecraft interference has been removed from the spin averaged magnetic field, a sensor offset still remains on the spin axis component. It is not possible to estimate the spin axis offset during the eclipse calibration analysis, and an independent method is required. In the case of TC-1, solar wind analysis is used to correct for the unknown spin axis offset. This method allows the calculation of the offset on a non-spinning sensor axis by using a natural property of the solar wind (Hedgecock, 1975). The solar wind is known to contain short period fluctuations which are predominantly rotational in nature. This means that there should be no correlation between the magnetic field magnitude and the magnetic field component that we are interested in, in this case the spin axis component, for a particular period of solar wind data. However, if there is a sensor offset in the component of interest then this introduces a correlation between the magnetic field in this component and the magnetic field magnitude. The offset is then determined by finding a field value that when removed from the magnetic field in the component of interest, results in zero correlation between the magnetic field magnitude and in this case the spin axis component. More precisely this method produces a correction to the actual spin axis offset and a correction to any error that there may have been during the removal of the spacecraft interference field. The success of this method relies on the selection of the best periods of solar wind data for this analysis to be performed; this means finding the periods of data which contain the highest proportion of rotational fluctuations. This selection has been done using methods developed for use with data from the four cluster spacecraft (private communication H. K. Schwarzl, K. Khurana, M. Kivelson, UCLA). The present best estimate of the spin axis offset correction to the spin averaged data which has had the interference removed using the dual magnetometer analysis is $+2.97 \pm 1.0 \mathrm{nT}$. This value is to be applied to the spin axis data in all ranges.

There are two remaining points to be made about the application of the methods described above for calibrating and removing spacecraft interference from TC-1 magnetic field data. Firstly, the eclipse calibration cannot provide an estimate for the spin axis offsets for either the inboard or outboard sensors. The uncertainty in these offsets is significant because the associated error is carried forward into the median $B_{i t}-B_{o t}$ calculations. In order to take account of this problem the inboard spin axis offset is shifted during the eclipse calibration stage until both the inboard and outboard magnetic field measurements are the same in the eclipse data 


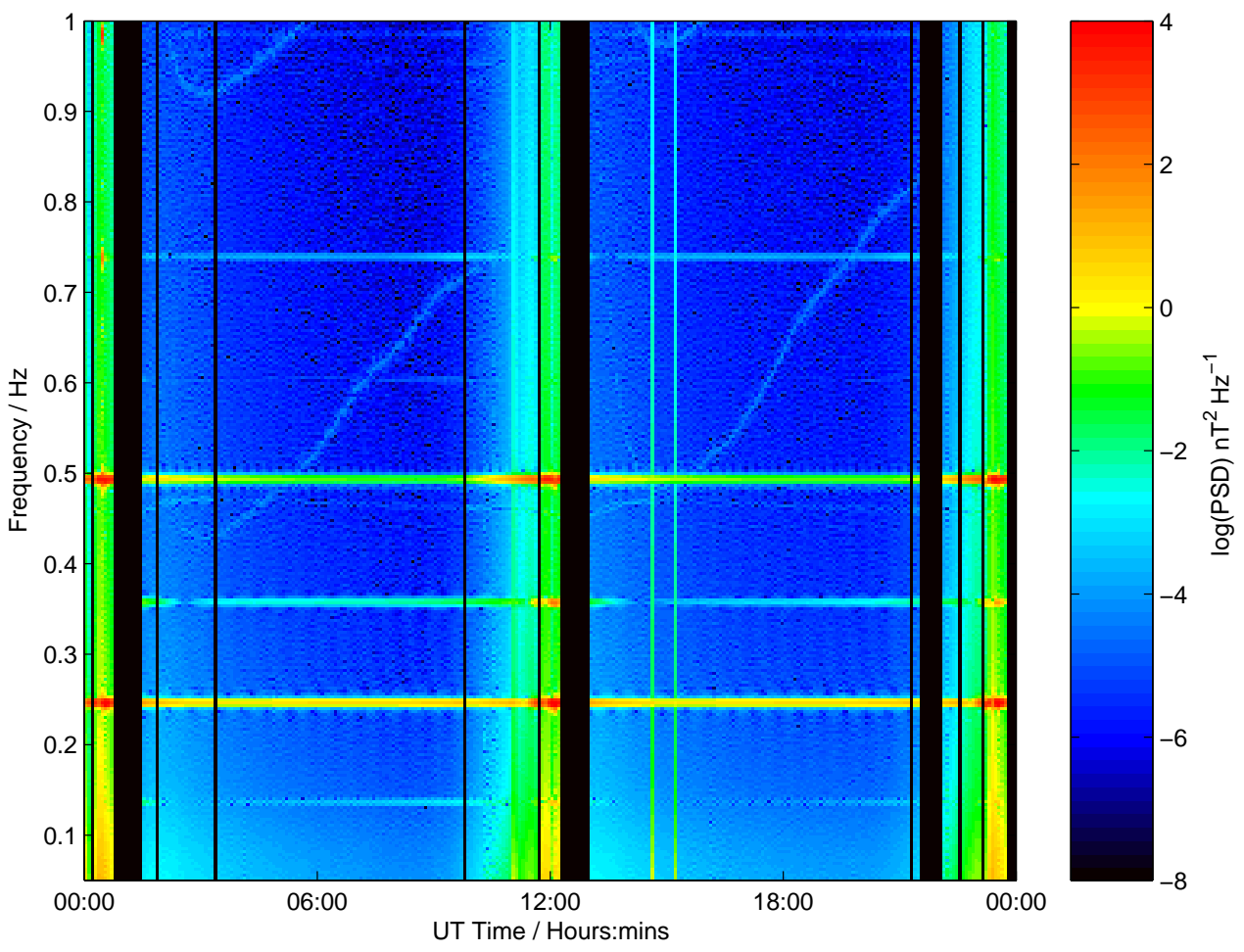

Fig. 12. A dynamic spectrum of the un-calibrated spin plane magnetic field magnitude using TC-2 data from 23 August 2004. The dynamic spectrum shows the power spectral density, which is represented by the colour scale, in the frequency range 0 to $1 \mathrm{~Hz}$ which is calculated using data bins of 5 min over the period of 1 day.

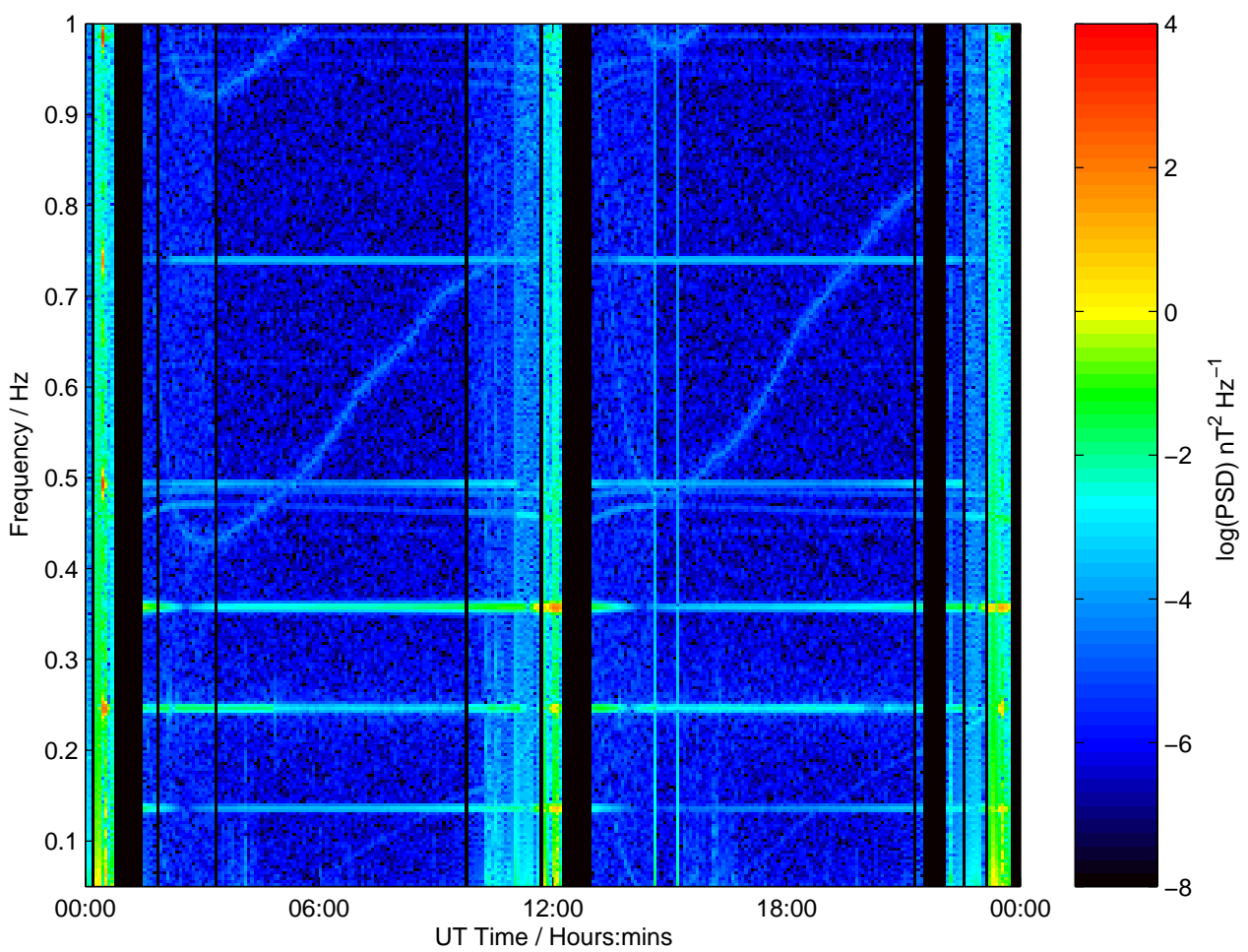

Fig. 13. A dynamic spectrum of the calibrated spin plane magnetic field magnitude using TC-2 data from 23 August 2004. The dynamic spectrum shows the power spectral density, which is represented by the colour scale, in the frequency range 0 to $1 \mathrm{~Hz}$ which is calculated using data bins of 5 min over the period of 1 day. 


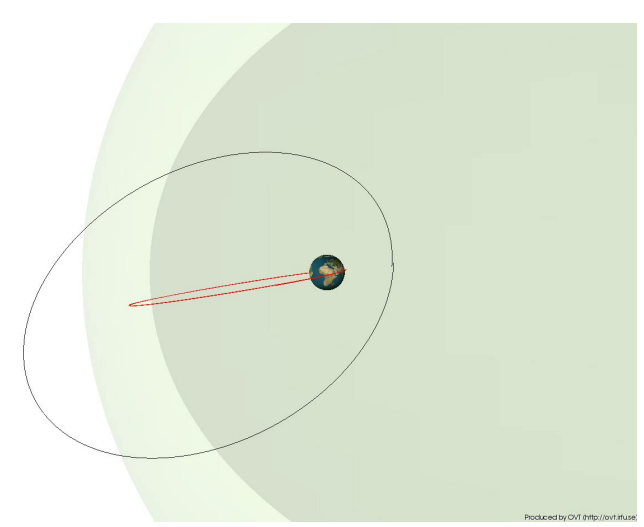

Fig. 14. Cluster (black) and TC-1 (red) orbits visualised in the GSE $\mathrm{x}-\mathrm{z}$ plane for approximately noon magnetic local time in February 2004.

where there is no spacecraft interference. The correction to the outboard spin axis offset can then be made as before using the solar wind analysis but now the uncertainty in the calculation of $B_{o s}$ due to spin axis offsets has been removed. Finally, the solar wind analysis must be performed in the SpacecraftSun (SCS) coordinate system so that the spin axis magnetic field direction is in direct alignment with the actual spacecraft spin axis. This means that the analysis to remove the spacecraft field is also performed in the SCS coordinate system and only after the spin axis offset correction has been made is the data transformed into a scientific coordinate system such as GSE and the production of the final data product is completed.

\subsection{TC-2}

Following the problems encountered with the spacecraft interference signal on TC- 1 a concerted effort was made to reduce the effect on TC-2 before its launch. A preliminary calibration analysis on TC-2 magnetic field data has shown that the spacecraft interference caused by the solar generator has been reduced by an order of magnitude. As a result it has been found that with care it is possible to estimate calibration parameters using standard techniques.

Figures 12 and 13 demonstrate that an initial calibration for 23 August 2004 interval has shown promising results. Figure 12 is a dynamic spectrum of the un-calibrated spin plane magnetic field magnitude binned using 5 min periods of data to perform the Fourier transform and done over the full day. The vertical axis shows that the calculations have been done in the frequency range 0 to $1 \mathrm{~Hz}$ and the horizontal axis shows the time. The colour scale refers to the log of the power spectral density. Figure 13 shows a similar dynamic spectrum, but in this case the data has been calibrated using the preliminary calibration performed on data from this day. By comparing Figs. 12 and 13 it can be seen that, in the case of the calibrated data, the power spectral density has been significantly reduced at approximately $0.25 \mathrm{~Hz}$ and approximately $0.5 \mathrm{~Hz}$ which is the spacecraft spin frequency and its 2 nd harmonic respectively. The power which remains in the dynamic spectrum of the calibrated data at these two frequencies is likely to be due to a combination of small errors in the calibration parameters and the spacecraft interference signal. Both Figs. 12 and 13 also show that there is spectral power in the magnetic field data at other frequencies which are not spacecraft spin harmonics. The source of these signals is not known at present and more work needs to be done to understand these signatures. As the power of these signals is not produced at spin harmonics they do not effect the determination of in-flight calibration parameters for TC-2.

\section{Highlights from the first year of instrument opera- tions}

TC-1 launched into the first dayside phase of the Double Star mission at the end of 2003. The FGM instrument started routine operations during January 2004. It was apparent that the revised orbit of TC-1, with an apogee of $13.4 R_{E}$ (caused by the over-performance of the launcher upper-stage), resulted in numerous excursions beyond the bow shock into un-disturbed solar wind. The intended orbit would have put the TC-1 apogee at $11.5 R_{E}$, such that at noon local-time (Fig. 14) the spacecraft would have been skimming the nominal location of the magnetopause at the sub-solar point. This valuable observing location is now lost, since a TC-1 orbit under typical solar wind conditions now takes the spacecraft through the magnetopause at local times earlier and later than noon. However, the solar wind observations have a number of advantages from the FGM point-of-view: firstly, the possibility to calibrate the sensors using statistical properties of the IMF (Hedgecock, 1975), and secondly the science opportunities raised through having combined Cluster/Double Star measurements of the bow shock and/or solar wind.

Simultaneous measurements from the Double Star and Cluster spacecraft provide the opportunity to study the large scale structure and variability of plasma phenomena such as the bow shock; magnetosheath waves and turbulence; and the magnetopause. An example of magnetic field data, from an extended period of bow shock crossings by TC-1, is shown in Fig. 15 (the data are shown in GSE coordinates). During this time, TC-1 travelled through the magnetosheath to apogee at 13.4 $R_{E}$ in the solar wind, at a local time of approximately 16:00, and then back into the magnetosheath, while the four Cluster spacecraft remained in the solar wind for the whole period. These data were taken during a period of high solar activity and a number of fast ICMEs passed the Earth: the data shown in Fig. 15 are from within one such ICME. Preliminary ACE SWEPAM data show a solar wind speed around $500 \mathrm{~km} / \mathrm{s}$ and an unusually high solar wind density at this time.

An expanded plot of one hour of magnetic field data is shown in Fig. 16. The data from TC-1 (shown in blue) are shifted forwards in time by $173 \mathrm{~s}$, to approximately synchronise them with the data recorded by Cluster (spacecraft number 2, shown in red). This shift reflects the fact that TC-1 was 

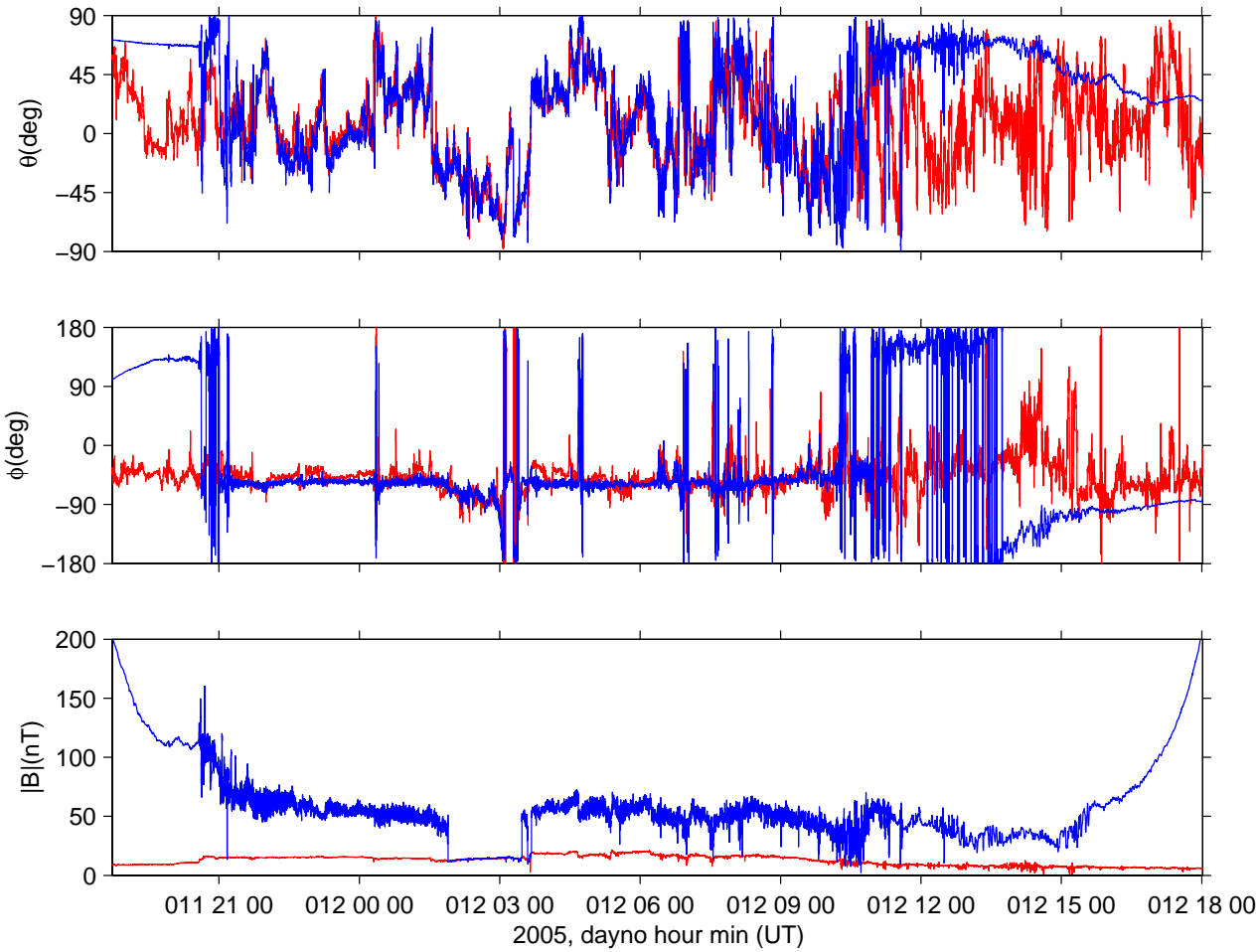

Fig. 15. Overview of Cluster spacecraft 2 (red) and Double Star TC-1 (blue) magnetic field data during one apogee pass by the TC-1 spacecraft on 12 January 2005 (GSE coordinates).
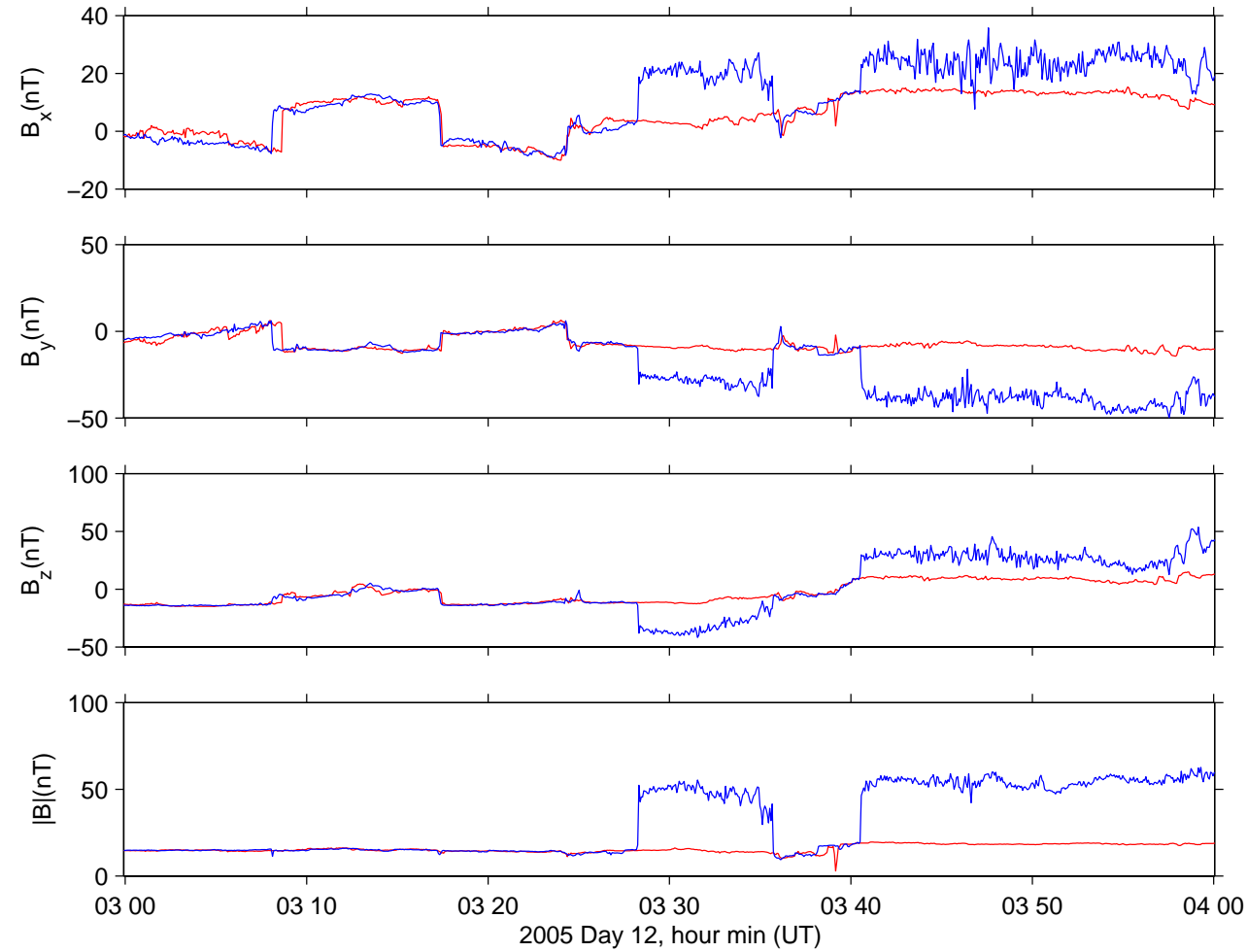

Fig. 16. One hour of magnetic field data (in GSE coordinates) from Cluster spacecraft 2 (red) and Double Star TC-1 (blue) on 12 January 2005. TC-1 encountered the bow shock at 03:28, 03:36 and 03:41. 


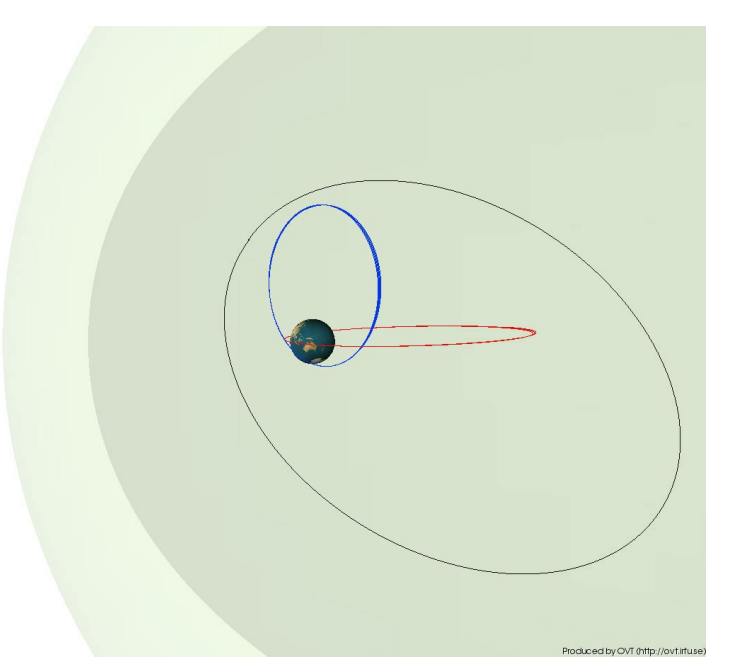

Fig. 17. Cluster (black), TC-1 (red) and TC-2 (blue) orbits visualised in the GSE $\mathrm{x}-\mathrm{z}$ plane for approximately midnight magnetic local time in August 2004.

downstream of the Cluster spacecraft, resulting in a convection delay: the spacecraft were separated by approximately 5.4 $R_{E}$ in the GSE x direction and 7.4 $R_{E}$ perpendicular to this. Several solar wind discontinuities are visible at both spacecraft in the first half of the interval as sharp changes in the magnetic field components. However, the relative times at which they passed Cluster and TC-1 are slightly different for different discontinuities, as a result of the different orientations of the discontinuity planes. Curvature of the discontinuities may also be important: by comparison of the local discontinuity orientation at the Cluster formation, determined by four-spacecraft timing, with the time at which the event passed TC-1, this curvature can be estimated. Comparison with additional spacecraft, such as Geotail, could allow an estimate of discontinuity curvature in three dimensions.

TC-1 encountered the bow shock three times during the second half of Fig. 16 while the Cluster spacecraft remained in the solar wind. Such encounters make it possible to study the dynamics of the shock motion in response to changes in upstream solar wind conditions. We note that there were at most small changes in the magnetic field measured at Cluster during the TC-1 bow shock encounters, reflecting the sensitivity of the bow shock's motion and location to small variations in upstream conditions.

One of the solar wind discontinuities in Fig. 16, that at 03:24, was accompanied at Cluster by high frequency ( $f \sim 1 \mathrm{~Hz}$ ) fluctuations consistent with generation by ions streaming along the discontinuity from the bow shock. Such an "active current sheet" might be a signature of a Hot Flow Anomaly (HFA: Schwartz et al., 1985; Lucek et al., 2004) at an early stage of evolution. TC-1, closer to the bow shock, observed no evidence of a developed HFA, perhaps reflecting the timescale over which waves are generated by streaming ions. Four-spacecraft Cluster data make it possible to determine the orientation and motion of the discontinuity

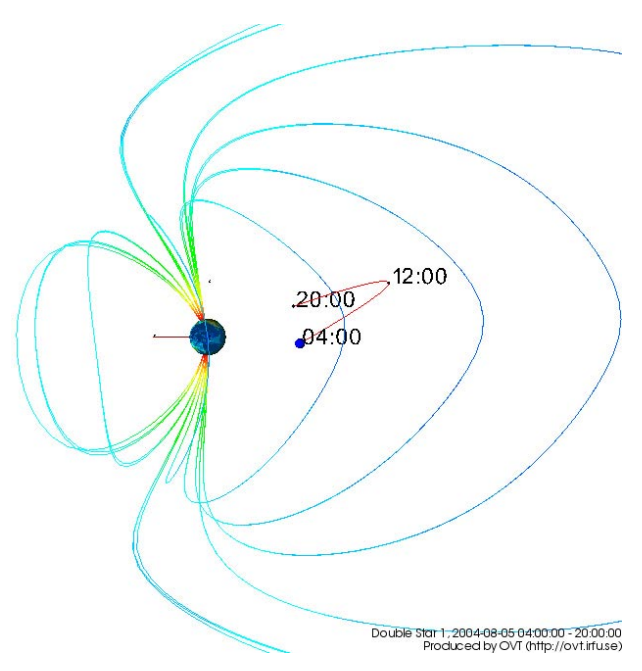

Fig. 18. TC-1 orbit in the GSM $x-z$ plane (viewed from the $+y$ direction) for the 5 August 2004, 04:00 to 20:00 UT.

associated with this event; TC-1, situated closer to the bow shock, gives a more widely separated observation of the same event. Cluster and TC-1 together, therefore, provide information about variations on two scales simultaneously. Further analysis of this and other similar events should improve our understanding of the large scale temporal and spatial evolution of HFAs and their associated signatures, both upstream of the bow shock and in the magnetosheath.

In July 2004, the TC-2 spacecraft was launched; the FGM instrument was commissioned in early August. From this time through to late autumn of that year, the six-spacecraft constellation was in its first night-side magnetosphere phase (Fig. 17). Figure 19 shows the TC-1 magnetic field observations on 5 August 2004 from 04:00 to 20:00 UT. At the apogee of its orbit $\left(13.4 \mathrm{R}_{E}\right)$, the spacecraft is travelling relatively slowly. Consequently, TC- 1 spends a considerable period of time near the inner edge of the current sheet. At 04:00 the spacecraft was in the Southern Hemisphere of the magnetosphere at a GSM position of $(-9.9,-1.6,-0.1) R_{E}$. Moving slowly dawnward and northward (Fig. 18) the spacecraft crossed the neutral sheet and entered into the Northern Hemisphere at approximately 07:00 UT. It stayed in the Northern Hemisphere until about 12:00 UT. From approximately 12:00 UT until 16:00 UT, we observe multiple crossings of the neutral sheet, as indicated by the reversal of the $\mathrm{B}_{x}$ polarity. These neutral sheet crossings are a consequence of a north-south "flapping" motion of the current sheet. In the same time interval, FGM measurements from the Cluster spacecraft, which were approximately $5 R_{E}$ tailward from TC-1, showed a very similar signature. Using four point magnetic field measurements from Cluster, it has been shown that these observations can be interpreted as a wave structure propagating from central sector of the magnetotail toward dawn direction (Zhang et al., 2005). Simultaneous observations by TC- 1 and Cluster have therefore provided a first global picture of this kind of wave structure. 

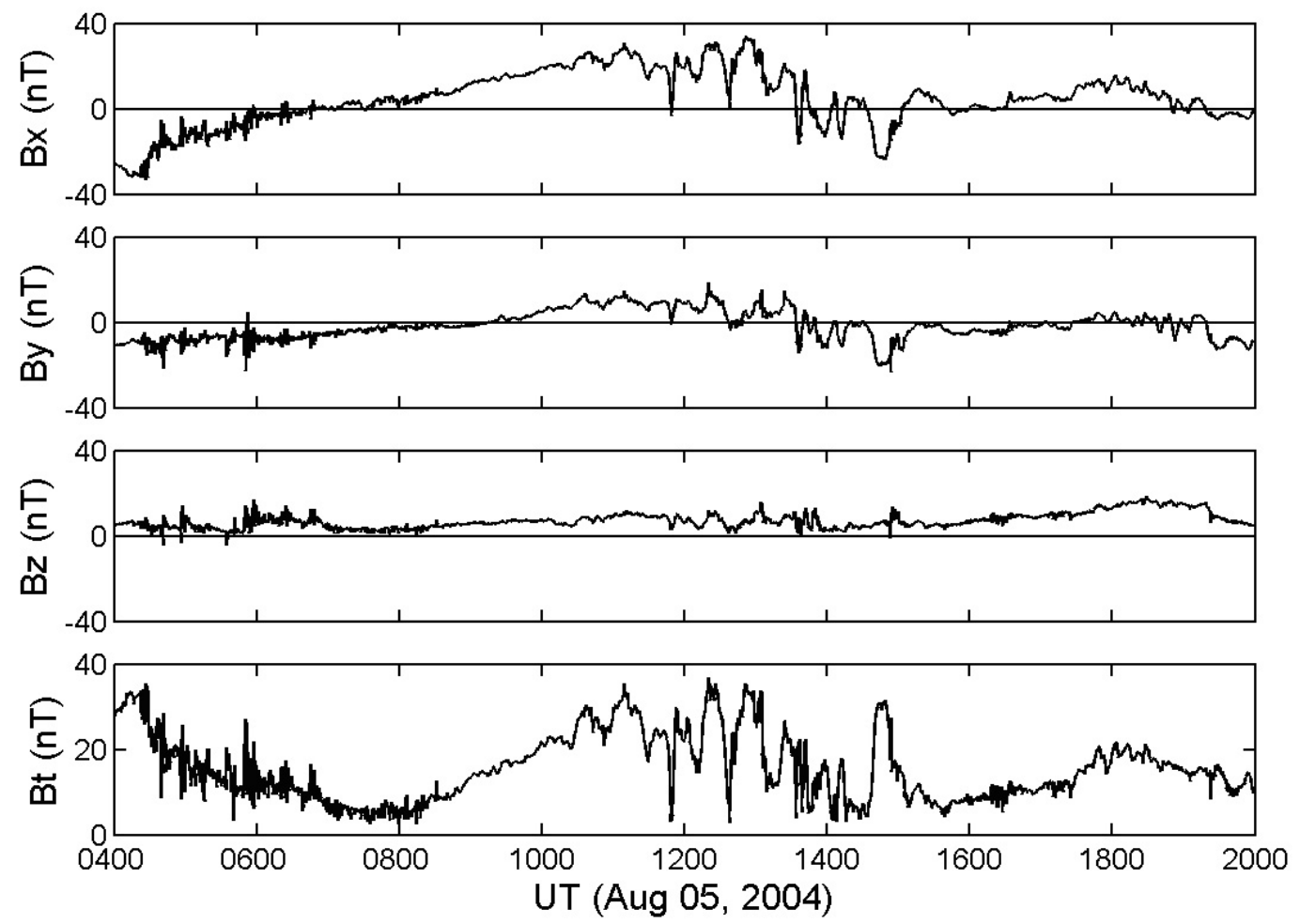

Fig. 19. TC-1 spin-resolution magnetic field data in GSM coordinates, 5 August 2004, 04:00 to 20:00 UT.

Acknowledgements. We are greatly indebted to the European Space Agency (ESA) and the Chinese National Space Administration (CNSA) who made the Double Star mission possible, and for all those at the Centre for Space Science and Applied Research (CSSAR) in Beijing who initiated and implemented the project, in particular Professors Zhenxing Liu and Ji Wu. We thank the ESA team for their tireless support and hard work on behalf of the European payload: B. Gramkow, P. Escoubet, P. Glaude and H. Laakso, and special thanks also to R. Nord and S. Osipenco at Astrium $\mathrm{GmbH}$. We are very grateful to the engineers of the China Academy of Space Technology, in particular S. Yuan who solved many interfacing problems, and for great help with spacecraft magnetics Y. Qi. Financial support for the construction of the magnetometers has been provided by ESA, and for the instrument operations by the UK Particle Physics and Astronomy Research Council.

ACE data were provided by the ACE Science Center.

Orbit plots are courtesy of the OVT team at IRF Uppsala, Sweden.

Topical Editor T. Pulkkinen thanks two referees for their help in evaluating this paper.

\section{References}

Acuña, M. H.: Fluxgate magnetometer for outer planets exploration, IEEE Trans. Magn., 10, 519-523, 1974.

Balogh., A., Cowley, S. W. H., Dunlop, M. W., et al.: The Cluster magnetic field investigation: Scientific objectives and instrumentation, in: Cluster: mission payload and supporting activities, ESA SP-1159, 95, 1993.

Balogh, A., Dunlop, M. W., Cowley, S. W. H., Southwood, D. J., Thomlinson, J. G., Glassmeier, K.-H., Musmann, G., Lühr, H.,
Buchert, S., Acuña, M. H., Fairfield, D. H., Slavin, J. A., Riedler, W., Schwingenschuh, K., and Kivelson, M. G.: The Cluster Magnetic Field Investigation, Space Sci. Rev., 79, 65, 1997.

Balogh, A., Carr, C. M., Acuña, M. H., Dunlop, M. W., T. Beek, T. J., Brown, P., Fornacon, K.-H., Georgescu, E., Glassmeier, K.H., Harris, J., Musmann, G., Oddy, T., and Schwingenschuh, K.: The Cluster magnetic field investigation: overview of in-flight performance and initial results, Ann. Geophys., 19, 1207-1217, 2001,

SRef-ID: 1432-0576/ag/2001-19-1207.

Fornacon, K.-H., Auster, H.-U., Georgescu, E., Baumjohann, W., Glassmeier, K.-H., Haerendel, G., Rustenbach, J., and Dunlop, M.: The magnetic field experiment onboard Equator-S and its scientific possibilities, Ann. Geophys., 17, 1521-1527, 1999,

SRef-ID: 1432-0576/ag/1999-17-1521

Geyger, W. A.: The ring core magnetometer - A new type of second harmonic fluxgate magnetometer, AIEE Trans., 81, 65-73, 1965.

Hedgecock, P. C.: A correlation technique for magnetometer zero level determination, Space Sci. Inst., 1, 83-90, 1975.

Kellock, S., Austin, P., Balogh, A., Gerlach, B., Marquedant, R., Musmann, G., Smith, E., Southwood, D., and Szalai, S.: Cassini dual technique magnetometer instrument (MAG), Proc. SPIE, Denver, Colorado, 2803, 141, 1996.

Liu, Z. X., Escoubet, C. P., Pu, Z., Laakso, H., Shi, J. K., Shen, C., and Hapgood, M.: The Double Star mission, Ann. Geophys., 23, 2707-2712, 2005.

Lucek, E. A., Horbury, T. S., Balogh, A., Dandouras, I., and Rème, H.: Cluster observations of hot flow anomalies, J. Geophys. Res., 109, A06207, doi:10.1029/2003JA010016, 2004.

Nakamura, R., Baumjohann, W., Zhang, T. L., Carr, C. M., Balogh, A., Glassmeier, K.-H., Rème, H., Dandouras, I., Takada, T., 
Volwerk, M., Asano, Y., Eichelberger, H.-U., Mouikis, C., Kistler, L. M., Klecker, B., and Amm, O.: Cluster and Double Star observations of dipolarization, Ann. Geophys., 23, 29152920, 2005.

Ness, N. F., Behannon, K. W., Lepping, R. P., and Schatten, K. H.: Use of two magnetometers for magnetic field measurements on a spacecraft, J. Geophys. Res., 76, 3565-3573, 1971.

Primdahl, F.: The fluxgate magnetometer, J. Phys. E. Sci. Instrum., 12, 241-253, 1979.

Pu, Z. Y., Xiao, C. J., Huang, Z. Y., Zhang, X. G., Fu, S. Y., Liu, Z. X., Dunlop, M. W., Zong, Q. G., Carr, C. M., Rème, H., Dandouras, I., Fazakerley, A., Phan, T., Zhang, T. L., Zhang, H., and Wang, X. G.: Double Star TC-1 observations of component reconnection at the dayside magnetopause: a preliminary study, Ann. Geophys., 23, 2889-2895, 2005.
Ripka, P.: Review of fluxgate sensors, Sensors and Actuators A, 33, 129-1411, 1992.

Schwartz, S. J., Chaloner, C. P., Hall, D. S., Johnstone, A. D., Rijnbeek, R. P., Southwood, D. J., and Wooliscroft, L. J. C.: An active current sheet in the solar wind, Nature, 318, 269-271, 1985.

Volwerk, M., Zhang, T. L., Glassmeier, K.-H., Nakamura, R., Runov, A., Baumjohann, W., Eichelberger, H.-U., Carr, C. M., Klecker, B., Balogh, A., and Reme, H.: Plasma flow channels with ULF waves observed by Cluster and Double Star, Ann. Geophys., 23, 2929-2935, 2005.

Zhang, T. L., Nakamura, R., Volwerk, M., Runov, A., Baumjohann, W., Eichelberger, H.-U., Carr, C. M., Balogh, A., Sergeev, V., Shi, J. K., and Fornacon, K.-H.: Double Star/Cluster observation of neutral sheet oscillations on 5 August 2004, Ann. Geophys., 23, 2909-2914, 2005. 